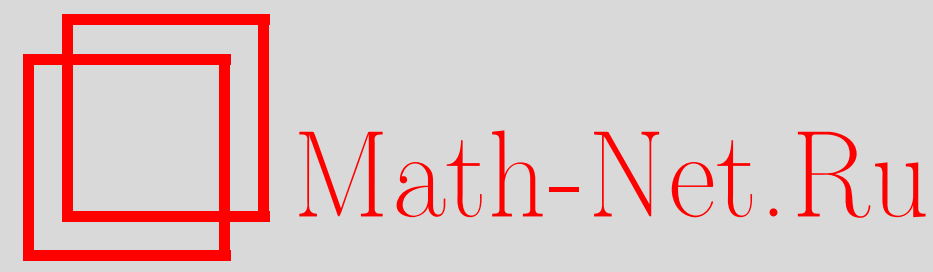

М. И. Вишик, В. В. Чепыжов, Аппроксимация траекторий, лежащих на глобальном аттракторе гиперболического уравнения с быстро осциллирующей по времени внешней силой, Матем. сб., 2003, том 194, номер 9, 3-30

DOI: https://doi.org/10.4213/sm765

Использование Общероссийского математического портала Math-Net.Ru подразумевает, что вы прочитали и согласны с пользовательским соглашением

http: //www.mathnet.ru/rus/agreement

Параметры загрузки:

IP : 3.93.64.190

26 апреля 2023 г., 14:38:40 


\section{Аппроксимация траекторий, лежащих на глобальном аттракторе гиперболического уравнения с быстро осциллируюшей по времени внешней силой}

\footnotetext{
Рассматривается квазилинейное диссипативное волновое уравнение при периодических граничных условиях с внешней силой $g(x, t / \varepsilon)$, быстро осциллирующей по $t$. Кроме того, предполагается, что при $\varepsilon \rightarrow 0+$ функция $g(x, t / \varepsilon)$ в слабом смысле (в $\left.L_{2, w}^{\text {loc }}\left(\mathbb{R}, L_{2}\left(\mathbb{T}^{n}\right)\right)\right)$ стремится к функции $\bar{g}(x)$, а усредненное волновое уравнение (с внешней силой $\bar{g}(x))$ имеет лиш конечное число стационарных точек $\left\{z_{i}(x), i=1, \ldots, N\right\}$, каждая из которых является гиперболической. Доказано, что глобальный аттрактор $\mathscr{A}_{\varepsilon}$ исходного уравнения отклоняется в энергетической норме от глобального аттрактора $\mathscr{A}_{0}$ усредненного уравнения на величину $C \varepsilon^{\rho}$, причем для $\rho$ дается явная формула. Кроме того, доказано, что любой кусок траектории $u^{\varepsilon}(t)$ исходного уравнения, лежащей на $\mathscr{A}_{\varepsilon}$ и временной длины $C \log (1 / \varepsilon)$, допускает аппроксимацию порядка $C_{1} \varepsilon^{\rho_{1}}$ с помощью конечного числа кусков траекторий, лежащих на неустойчивых многообразиях $M^{u}\left(z_{i}\right)$ усредненного уравнения. Для $\rho_{1}$ дается явное выражение.

Библиография: 14 названий.
}

Рассматривается следующее модельное неавтономное диссипативное гиперболическое уравнение:

$$
\partial_{t}^{2} u+\gamma \partial_{t} u=\Delta u-\delta^{2} u-f(u)+g\left(x, \frac{t}{\varepsilon}\right)
$$

при периодических граничных условиях: $x \in \mathbb{T}^{n}$. Здесь $\delta^{2}>0, \gamma>0,|f(u)| \leqslant$ $C\left(|u|^{\rho+1}+1\right)$, где $\rho<2 /(n-2)$ при $n \geqslant 3$ и $\rho-$ любое число при $n=1,2$. Кроме того, $f(u)$ удовлетворяет некоторым дополнительным условиям, которые сформулированы в $\S 2$. Функция $g(x, z)$ - трансляционно компактная (тр.к.) в пространстве $L_{2}^{\text {loc }}(\mathbb{R}, H), H=L_{2}\left(\mathbb{T}^{n}\right)$. Это означает, что множество сдвигов $\{g(x, z+h), h \in \mathbb{R}\}$ образует предкомпактное множество в $L_{2}([-T, T], H)$ при любом фиксированном $T \in \mathbb{R}_{+}$. Отметим, что тр.к. являются функции $g(x, z)$, периодические по $z$ со значениями в $H$, квазипериодические и почти периодические функции $g(x, z)$ по $z$ со значениями в $H$. Однако класс тр.к. функций $g(x, z)$ является значительно более широким (см. [1]).

Уравнения вида (0.1) встречаются в релятивистской квантовой механике (см., например, [2] и приведенную там литературу).

Предполагается, что при $\varepsilon \rightarrow 0+$ функция $g(x, t / \varepsilon) \rightarrow \bar{g}(x)$ в $L_{2, w}^{\text {loc }}(\mathbb{R}, H)($ см. $\S 2)$. Функция $\bar{g}(x)$ называется усреднением функиии $g(x, t / \varepsilon)$.

Наряду с уравнением (0.1) рассматривается усредненное уравнение

$$
\partial_{t}^{2} \bar{u}+\gamma \partial_{t} \bar{u}=\Delta \bar{u}-\delta^{2} \bar{u}-f(\bar{u})+\bar{g}(x), \quad x \in \mathbb{T}^{n} .
$$


Уравнение (0.2) обладает глобальной функцией Ляпунова:

$$
\begin{gathered}
\mathscr{L}(\bar{u})=\int_{\Omega}\left(\frac{1}{2}\left|\partial_{t} \bar{u}(\cdot, t)\right|^{2}+\frac{1}{2}|\nabla \bar{u}(\cdot, t)|^{2}+\frac{\delta^{2}}{2}|\bar{u}|^{2}+F(\bar{u})-\bar{g}(\cdot) \bar{u}(\cdot, t)\right) d x, \\
F(\bar{u})=\int_{0}^{\bar{u}} f(v) d v .
\end{gathered}
$$

Предполагается, что уравнение (0.2) имеет конечное число стационарных точек $\left\{z_{i}(x), i=1, \ldots, N\right\}$ :

$$
\Delta z_{i}-\delta^{2} z_{i}-f\left(z_{i}\right)+\bar{g}(x)=0, \quad x \in \mathbb{T}^{n}
$$

и притом каждая из них является гиперболической (см. [3]-[5]). Тогда, как показано, например, в [3], [4], глобальный аттрактор $\overline{\mathscr{A}}$ уравнения $(0.2)$ имеет следующую структуру:

$$
\overline{\mathscr{A}}=\bigcup_{i=1}^{N} M^{u}\left(z_{i}\right),
$$

где $M^{u}\left(z_{i}\right)$ - полное неустойчивое многообразие, проходящее через точку $z_{i}$.

Через $H_{s}, s \in \mathbb{R}$, как обычно, обозначается шкала пространств Соболева, где $H_{0}=H=L_{2}\left(\mathbb{T}^{n}\right), H_{1}=H_{1}\left(\mathbb{T}^{n}\right)$.

Сформулируем основной результат, полученный в настоящей статье. Пусть $\left\{u^{\varepsilon}(x, t), t \in \mathbb{R}\right\}$ - любая полная траектория уравнения (0.1), ограниченная в энергетическом пространстве $E:=H_{1} \times H$. Рассмотрим кусок этой траектории временной длины $r_{\varkappa}(\varepsilon)=(\varkappa /((\rho+\nu) 2(2+\varkappa))) \log (1 / \varepsilon), 0<\varkappa<+\infty:\left\{u^{\varepsilon}(x, t)\right.$, $\left.\theta \leqslant t \leqslant \theta+r_{\varkappa}(\varepsilon)\right\}, \theta \in \mathbb{R}$. Здесь $\nu$ - коэффициент экспоненциального притяжения траекторий $\bar{u}(x, t)$ уравнения $(0.2)$ его глобальным аттрактором $\overline{\mathscr{A}}$. Число $\rho$ - коэффициент экспоненциального расхождения траекторий уравнения (0.2) от траекторий уравнения (0.1) при одинаковых начальных условиях (см. §3). Число $\varkappa$ - любое фиксированное положительное число. Тогда найдется такая конечномерная составная траектория $\widetilde{u}^{0} \in \bigcup_{i=1}^{N} M^{u}\left(z_{i}\right)($ см. $\S 4)$, что

$$
\begin{gathered}
\left\|\left(u^{\varepsilon}(\cdot, t), \partial_{t} u^{\varepsilon}(\cdot, t)\right)-\left(\widetilde{u}^{0}(\cdot, t), \partial_{t} \widetilde{u}^{0}(\cdot, t)\right)\right\|_{E} \leqslant C \varepsilon^{\alpha \varkappa}, \\
\alpha_{\varkappa}=\frac{\nu}{(2+\varkappa)(\rho+\nu)}, \quad \theta \leqslant t \leqslant \theta+r_{\varkappa}(\varepsilon),
\end{gathered}
$$

причем $C$ не зависит от $u^{\varepsilon}$ и $\theta$. Таким образом, грубо говоря, куски полных траекторий $u^{\varepsilon}(x, t)$ уравнения $(0.1)$, имеющие временную длину, пропорциональную $\log (1 / \varepsilon)$, могут быть аппроксимированы с помощью нескольких кусков траекторий усредненного уравнения $(0.2)$, лежащих на $\bigcup_{i=1}^{N} M^{u}\left(z_{i}\right)$. При этом $(0.4)$ дает равномерную оценку сверху их отклонений в метрике $E$.

Для доказательства этого результата в первых параграфах приводятся необходимые сведения и результаты. В $\S 1$ дается определение глобального аттрактора $\mathscr{A}$ неавтономного эволюционного уравнения

$$
\partial_{t} u=L(u, t)
$$


Кроме того, приводится определение ядра $\mathscr{K}$ этого уравнения: $\mathscr{K}$ является объединением всех ограниченных полных траекторий уравнения (0.5). Для уравнения

$$
\partial_{t} u=L(u)+g_{0}(x, t)
$$

с тр.к. функцией $g_{0}(x, t) \in L_{2}^{\text {loc }}(\mathbb{R}, H)$ приводится формула, описывающая структуру глобального аттрактора $\mathscr{A}$ уравнения (0.6).

В $\S 2$ изучается уравнение (0.1) с быстро осциллирующей внешней силой $g(x, t / \varepsilon)$, причем $g(\cdot, z)$ - тр.к. функция в $L_{2}^{\mathrm{loc}}\left(\mathbb{R}, H_{\sigma_{2}}\right)$, a $g_{x_{i}}^{\prime}(\cdot, z), i=1, \ldots, N,-$ тр.к. функция в $L_{2}^{\text {loc }}\left(\mathbb{R}, H_{\sigma_{2}-1}\right)$, где $\sigma_{2}=1-2 \rho /(n-2)$ (напомним, что $(\rho+1)-$ порядок роста $f(u)$ по $u$ ) (см. $\S 2)$. При выполнении этих условий доказывается, что уравнение $(0.1)$ обладает равномерным глобальным аттрактором $\mathscr{A}_{\varepsilon}$ в энергетическом пространстве $E$.

В $\S 3$ исследуется отклонение $w=u^{\varepsilon}-u^{0}$, где $u^{\varepsilon}-$ решение уравнения $(0.1)$, $u^{0}$ - решение уравнения $(0.2)$, удовлетворяющие одинаковым начальным условиям. При этом налагается дополнительное условие на разность $g(x, t / \varepsilon)-\bar{g}(x):=$ $\widetilde{g}(x, t / \varepsilon)$. Предполагается, что существует первообразная $\widetilde{G}(\cdot, z)$ функции $\widetilde{g}(x, z)$ по $z$, удовлетворяющая следуюшему условию:

$$
\|\widetilde{G}(\cdot, z)\|_{H_{1}} \leqslant M \quad \forall z \in \mathbb{R}, \quad \partial_{z} \widetilde{G}(x, z)=\widetilde{g}(x, z) .
$$

Отметим, что в случае квазипериодической по $z$ функции $\widetilde{g}(x, z)$ это условие сводится к выполнению диофантова условия частотами функции $\widetilde{g}(x, z)$.

При выполнении этого условия доказана следующая оценка:

$$
\begin{gathered}
\left\|\left(w, \partial_{t} w\right)\right\|_{E}:=\left(\|w(\cdot, \tau+t)\|_{H_{1}}^{2}+\left\|\partial_{t} w(\cdot, \tau+t)\right\|_{H}^{2}\right)^{1 / 2} \leqslant C \varepsilon^{1 / 2} e^{\rho t} \\
\forall t \geqslant 0, \quad \forall \tau \in \mathbb{R} .
\end{gathered}
$$

Из указанных выше фактов в $\oint 4$ выводится аппроксимация (0.4) траекторий $u^{\varepsilon}(x, t)$ с помощью траекторий $\widetilde{u}^{0}(x, t) \in \bigcup_{i=1}^{N} M^{u}\left(z_{i}\right)$.

В $\S 4$ дается также оценка отклонения глобальных аттракторов $\mathscr{A}_{\varepsilon}$ от аттрактора $\mathscr{A}_{0}=\overline{\mathscr{A}}$ :

$$
\operatorname{dist}_{E}\left(\mathscr{A}_{\varepsilon}, \mathscr{A}_{0}\right) \leqslant C \varepsilon^{\nu /(2(\rho+\nu))} .
$$

Для неавтономных уравнений типа сайн-Гордона оценка вида (0.8) получена в $[6]$.

Для гиперболических уравнений с коэффициентами, быстро осциллирующими по $x$ (например, $g=g(x, x / \varepsilon)$ ), оценки вида (0.8) получены в [7]. Для более общих, чем (0.1), гиперболических уравнений с быстро осциллирующими по $x$ членами доказана лишь сходимость $\mathscr{A}_{\varepsilon}$ к $\mathscr{A}_{0}$ в соответствующих функциональных пространствах [1], [8], [9]. 


\section{§1. Глобальный аттрактор неавтономного эволюционного уравнения}

1.1. Рассматривается неавтономное эволюционное уравнение вида:

$$
\partial_{t} u=L(u, t), \quad t \geqslant \tau, \quad \tau \in \mathbb{R} .
$$

Здесь $L(u, t)$ - нелинейный оператор $L(\cdot, t): E_{1} \rightarrow E_{0}$ для любого $t \in \mathbb{R}$, где $E_{1}$ и $E_{0}$ - банаховы пространства такие, что $E_{1} \subseteq E_{0}$. Изучаются решения $u(t)$ уравнения (1.1), определенные при $t \geqslant \tau$. При $t=\tau$ задается начальное условие:

$$
\left.u\right|_{t=\tau}=u(\tau):=u_{\tau}, \quad u_{\tau} \in E
$$

где $E$ - банахово пространство такое, что $E_{1} \subseteq E \subseteq E_{0}$. Предполагается, что для любого $\tau \in \mathbb{R}$ и для любого $u_{\tau} \in E$ задача Коши (1.1), (1.2) имеет, и притом единственное, решение $u(t)$ такое, что $u(t) \in E$ для любых $t \geqslant \tau$. При этом в каждом конкретном случае объясняется, в каком смысле $u(t)$ удовлетворяет уравнению (1.1).

Пусть $\{U(t, \tau)\}, t \geqslant \tau, \tau \in \mathbb{R}$, следующее двупараметрическое семейство операторов, порожденное задачей $(1.1),(1.2)$, действующих в пространстве $E$ по формуле

$$
U(t, \tau) u_{\tau}=u(t), \quad t \geqslant \tau, \quad \tau \in \mathbb{R},
$$

где $u(t)$ - решение задачи (1.1), (1.2). Семейство операторов $\{U(t, \tau)\}$ обладает следующими свойствами:

1) $U(\tau, \tau)=\mathrm{Id}$ для любого $\tau \in \mathbb{R}$;

2) $U(t, s) \circ U(s, \tau)=U(t, \tau)$ для любых $t \geqslant s \geqslant \tau, \tau \in \mathbb{R}$.

Семейство операторов $\{U(t, \tau)\}$ называется прочессом, порожденным задачей (1.1), (1.2).

Для определения глобального аттрактора $\mathscr{A}$ процесса $\{U(t, \tau)\}$ введем сначала некоторые необходимые для этого понятия. Через $\mathscr{B}(E)$ обозначается семейство всех ограниченных множеств в $E$. Множество $B_{0}$ называется поглощающим, если для любого $B \in \mathscr{B}(E)$ существует число $h=h(B)$ такое, что

$$
U(t, \tau) B \subset B_{0} \quad \forall t, \tau, t-\tau \geqslant h .
$$

Множество $P \subset E$ называется притягивающим для процесса $\{U(t, \tau)\}$, если для любого $\varepsilon>0$ множество $\mathscr{O}_{\varepsilon}(P)$ является поглощающим для этого процесса. (Здесь и ниже через $\mathscr{O}_{\varepsilon}(M)$ обозначается $\varepsilon$-окрестность множества $M$ в пространстве $E$.) Очевидно, множество $P$ является притягивающим тогда и только тогда, когда для любого $B \in \mathscr{B}(E)$

$$
\sup _{\tau \in \mathbb{R}} \operatorname{dist}_{E}(U(\tau+h, \tau) B, P) \rightarrow 0, \quad h \rightarrow+\infty .
$$

Здесь $\operatorname{dist}_{E}(X, Y)$ понимается в смысле Хаусдорфа:

$$
\operatorname{dist}_{E}(X, Y)=\sup _{x \in X} \inf _{y \in Y}\|x-y\|_{E}
$$


ОПРЕДЕЛЕНИЕ 1.1. Множество $\mathscr{A} \subset E$ является глобальным аттрактором nроцесса $\{U(t, \tau)\}$, если оно замкнутое притягивающее множество этого процесса и обладает следующим свойством минимальности: оно принадлежит любому замкнутому притягиваюшему множеству $\mathscr{A}^{\prime}$ процесса $U(t, \tau): \mathscr{A} \subseteq \mathscr{A}^{\prime}$.

Это понятие глобального аттрактора $\mathscr{A}$ процесса $\{U(t, \tau)\}$ было введено в [10], [11], [1]. Процесс $\{U(t, \tau)\}$, обладающий компактным поглощающим множеством, называется компактным процессом. Процесс $\{U(t, \tau)\}$, обладаюший замкнутым притягивающим множеством, называется асимптотически компактным прочессом.

TЕОРема 1.1. Если прочесс $\{U(t, \tau)\}$ является асимптотически компактныцм, то он обладает компактныц в $Е$ глобальныцм аттрактором $\mathscr{A}$.

Доказательство этой теоремы дано в [1]. Там же приведена следуюшая формула для указанного в теореме 1.1 глобального аттрактора:

$$
\mathscr{A}=\omega(P):=\bigcap_{h \geqslant 0}\left[\bigcup_{t-\tau \geqslant h} U(t, \tau) P\right]_{E},
$$

где $P$ является любым притягиваюшим множеством процесса $\{U(t, \tau)\},[B]_{E}-$ замыкание множества $B$ в $E$.

1.2. Введем теперь понятие ядра процесса $\{U(t, \tau)\}$.

Функция $u(s), s \in \mathbb{R}$, со значениями в $E$ называется полной траекторией прочесса $U(t, \tau)$, если

$$
U(t, \tau) u(\tau)=u(t) \quad \forall t \geqslant \tau, \quad \tau \in \mathbb{R} .
$$

Полная траектория называется ограниченной, если множество $\{u(s), s \in \mathbb{R}\}$ ограничено в $E$.

ОПРЕДЕЛЕНИЕ 1.2. Множество всех ограниченных полных траекторий $\{u(t)$, $t \in \mathbb{R}\}$ процесса $\{U(t, \tau)\}$ назьвается его ядром и обозначается буквой $\mathscr{K}:$

$$
\mathscr{K}=\left\{u(\cdot) \mid u(t), t \in \mathbb{R}, \text { удовлетворяет (1.7) и }\|u(s)\|_{E} \leqslant C_{u} \forall s \in \mathbb{R}\right\} .
$$

Множество

$$
\mathscr{K}(t)=\{u(t) \mid u(\cdot) \in \mathscr{K}\}, \quad t \in \mathbb{R}
$$

называется сечением ядра в момент $t$.

ПРЕДЛОЖЕНИЕ 1.1. Если процесс $\{U(t, \tau)\}$ обладает глобальным аттрактором $\mathscr{A}$, то

$$
\bigcup_{t \in \mathbb{R}} \mathscr{K}(t) \subseteq \mathscr{A}
$$

(cм. [1]).

Отметим, что, как правило, включение в (1.8) является строгим, т.е. существуют точки глобального аттрактора $\mathscr{A}$, которые не являются значениями полных, ограниченных траекторий данного процесса $\{U(t, \tau)\}$. Ниже на конкретных примерах будет показано на каких полных траекториях "близких процессов" такие точки лежат. В весьма обшем случае процессов уравнений вида (1.1) эта проблема 
изучена в [1]. Ниже мы ограничимся выяснением этого вопроса для неавтономного эволюционного уравнения вида

$$
\partial_{t} u-L(u)=g_{0}(x, t)
$$

и соответствуюшего ему процесса $\{U(t, \tau)\}$. В дальнейшем изучаются лишш уравнения вида $(1.9)$, причем $g_{0}(x, t) \in L_{2}^{\text {loc }}(\mathbb{R}, H)$ (или $g_{0}(x, t) \in C^{\text {loc }}(\mathbb{R}, H)$ или другому аналогичного типа пространству). Здесь $H$ обозначает гильбертово или банахово пространство.

Напомним, что $g_{0}(x, t) \in L_{2}^{\text {loc }}(\mathbb{R}, H)$, если $g_{0}(x, t) \in L_{2}([-T, T], H)$ для любого $T>0$. Последовательность $\left\{g_{0}\left(x, t+h_{n}\right)\right\}, h_{n} \in \mathbb{R}$, сходится при $n \rightarrow+\infty \mathrm{k}$ функции $g(x, t)$ в $L_{2}^{\text {loc }}(\mathbb{R}, H)$, если она сходится к $g(x, t)$ в $L_{2}([-T, T], H)$ для любого $T>0$.

ОПРЕДЕЛЕНИЕ 1.3. Множество

$$
\mathscr{H}\left(g_{0}\right)=\left[\left\{g_{0}(x, t+h), h \in \mathbb{R}\right\}\right]_{L_{2}^{\text {loc }}}
$$

называется оболочкой функции $g_{0}(x, t)$ в $L_{2}^{\text {loc }} . \mathrm{B}(1.10)[\cdot]_{L_{2}^{\text {loc }}}$ означает замњкание в топологическом пространстве $L_{2}^{\text {loc }}(\mathbb{R}, H):=L_{2}^{\text {loc }}$.

ОПРЕДЕЛЕНИЕ 1.4. Функция $g_{0}(x, t)$ называется трансляционно компактной (тр.к.), если ее оболочка $\mathscr{H}\left(g_{0}\right)$ является компактным множеством в $L_{2}^{\text {loc }}$.

Аналогично определяется оболочка $\mathscr{H}\left(g_{0}\right)$ и тр.к. функция $g_{0}(x, t)$ в $C(\mathbb{R}, H)$.

Рассмотрим некоторые примеры тр.к. функций.

ПримеР 1. Пространство $L_{2}^{\text {loc }}(\mathbb{R}, H)$ состоит из функций $\xi(t), t \in \mathbb{R}$, со значениями в $H$, квадрат нормы которых локально интегрируем в смысле Бохнера:

$$
\int_{t_{1}}^{t_{2}}\|\xi(t)\|_{H}^{2} d t<+\infty \quad \forall\left[t_{1}, t_{2}\right] \subset \mathbb{R} .
$$

Последовательность $\left\{\xi_{n}(t)\right\}$ сходится к $\xi(t), n \rightarrow+\infty$, в $L_{2}^{\text {loc }}(\mathbb{R}, H)$, если

$$
\int_{t_{1}}^{t_{2}}\left\|\xi_{n}(t)-\xi(t)\right\|_{H}^{2} d t \rightarrow 0, \quad n \rightarrow \infty
$$

для любого интервала $\left[t_{1}, t_{2}\right] \subset \mathbb{R}$. Приведем критерий тр.к. функции $g_{0}(x, t) \in$ $L_{2}^{\text {loc }}(\mathbb{R}, H)$ (см., например, [1]).

Функция $g_{0}(t)$ со значениями в $H$ тр.к. в $L_{2}^{\text {loc }}(\mathbb{R}, H)$ тогда и только тогда, когда:

1) для любого $h \geqslant 0\left\{\int_{t}^{t+h} g_{0}(s) d s, t \in \mathbb{R}\right\}$ - предкомпактное множество в $H$;

2) сушествует положительная функция $\beta(s)>0, \beta(s) \rightarrow 0, s \rightarrow 0+$, такая, что

$$
\int_{t}^{t+1}\left\|g_{0}(s)-g_{0}(s+l)\right\|_{H}^{2} d s \leqslant \beta(|l|) \quad \forall t \in \mathbb{R} .
$$


Заметим, что для любой тр.к. функции $g_{0}$ в $L_{2}^{\text {loc }}(\mathbb{R}, H)$

$$
\sup _{t \in \mathbb{R}} \int_{t}^{t+1}\left\|g_{0}(s)\right\|_{H}^{2} d s<+\infty
$$

(cM. [1]).

Пример 2. Аналогично определяется тр.к. функция в пространстве $C(\mathbb{R}, H)$. Критерий тр.к. функции $g_{0} \in C(\mathbb{R}, H)$ аналогичен (с очевидньми изменениями) критерию, приведенному в примере 1 (см. [1]).

ПримеР 3. Почти периодическая (п.п.) функция $g_{0}(t)$ со значениями в $H$ является тр.к. функцией в $C_{b}(\mathbb{R}, H)$. Отметим, что в доказательстве этого факта используется критерий Бохнера-Амерно п.п. функции в $C_{b}(\mathbb{R}, H)($ см. [1], [12]). Подклассом п.п. функции являются квазипериодические функции. Функция $g_{0}(t) \in$ $C_{b}(\mathbb{R}, H)$ является квазипериодической (к.п.), если она представима в виде

$$
g_{0}(t)=\Phi\left(\alpha_{1} t, \ldots, \alpha_{k} t\right)=\Phi(\bar{\alpha} t), \quad \bar{\alpha}=\left(\alpha_{1}, \ldots, \alpha_{k}\right)
$$

причем функция $\Phi(\bar{\omega})=\Phi\left(\omega_{1}, \ldots, \omega_{k}\right)$ непрерывная и $2 \pi$-периодическая по $\omega_{i} \in \mathbb{R}$, $i=1, \ldots, k$ :

$$
\Phi\left(\omega_{1}, \ldots, \omega_{i}+2 \pi, \ldots, \omega_{k}\right)=\Phi\left(\omega_{1}, \ldots, \omega_{i}, \ldots, \omega_{k}\right), \quad i=1, \ldots, k
$$

Через $\mathbb{T}^{k}=[\mathbb{R} \bmod 2 \pi]^{k}$ обозначим $k$-мерный тор. Тогда $\Phi \in C\left(\mathbb{T}^{k}, H\right)$. Предполагается, что числа $\alpha_{1}, \ldots, \alpha_{k}$ в (1.12) рационально независимы. Легко доказать, что оболочка $\mathscr{H}\left(g_{0}\right)$ к.п. функции $g_{0}(t)$ в $C_{b}(\mathbb{R}, H)$ совпадает со следующим множеством функций:

$$
\left\{g_{0}\left(\bar{\alpha} t+\bar{\omega}_{0}\right) \mid \bar{\omega}_{0} \in \mathbb{T}^{k}\right\}=\mathscr{H}\left(g_{0}\right) .
$$

Следовательно, $\mathscr{H}\left(g_{0}\right)$ является непрерывным образом $k$-мерного тора $\mathbb{T}^{k}$.

В [1] приведены и другие примеры тр.к. функций.

1.3. Рассмотрим теперь семейство уравнений вида

$$
\partial_{t} u=L(u)+g(x, t)
$$

где $g(x, t)$ - любая функция из оболочки $\mathscr{H}\left(g_{0}(x, t)\right)$ в $L_{2}^{\text {loc }}(\mathbb{R}, H)$. Предполагается, что $g_{0}(x, t)$ - тр.к. функция в $L_{2}^{\text {loc }}(\mathbb{R}, H)$ (или $\left.C(\mathbb{R}, H)\right)$. Пусть задача Коши (1.14), (1.2) имеет, и притом единственное, решение при любых $\tau \in \mathbb{R}$ и $u_{\tau} \in E$, причем $u(t) \in E$ при всех $t \geqslant \tau$. Следовательно, определено семейство процессов $\left\{U_{g}(t, \tau)\right.$, $\left.g \in \mathscr{H}\left(g_{0}\right)\right\}$, действующих в $E$ и соответствующих задаче $(1.14),(1.2)$. Аналогично определению 1.1 дается определение равномерного (по $\left.g \in \mathscr{H}\left(g_{0}\right)\right)$ глобального аттрактора семейства процессов $\left\{U_{g}(t, \tau), g \in \mathscr{H}\left(g_{0}\right)\right\}$. 
ТЕОРема 1.2. Предположим, что функиия $g_{0}(x, t)-$ тр.к. в $L_{2}^{\operatorname{loc}}(\mathbb{R}, H)$. Пусть прочесс $\left\{U_{g_{0}}(t, \tau)\right\}$ асимптотически компактен в $E$, и пусть семейство процессов $\left\{U_{g}(t, \tau), g \in \mathscr{H}\left(g_{0}\right)\right\}$ непрерьвно в $E \times \mathscr{H}\left(g_{0}\right)$, m.e. $U_{g}(t, \tau) u_{\tau}$ непрерывно по $\left(u_{\tau}, g\right)$, принимая значения в $E$.

Тогда существует равномерный (относительно $g \in \mathscr{H}\left(g_{0}\right)$ ) глобальный аттрактор $\mathscr{A}$ семейства процессов $\left\{U_{g}(t, \tau)\right\}$. Аттрактор $\mathscr{A}$ допускает следующее представление:

$$
\mathscr{A}=\bigcup_{g \in \mathscr{H}\left(g_{0}\right)} \mathscr{K}_{g}(0)=\bigcup_{g \in \mathscr{H}\left(g_{0}\right)} \mathscr{K}_{g}(t), \quad t \in \mathbb{R},
$$

где $\mathscr{K}_{g}$ - ядро прочесса $U_{g}(t, \tau)$. Здесь $t \in \mathbb{R}-$ фиксированное число. Ядро $\mathscr{K}_{g}$ является непустым мнохеством при любом $g \in \mathscr{H}\left(g_{0}\right)$.

Доказательство теоремы 1.2 дано в [1] и [11].

Предположим, что множество $\mathscr{P},[\mathscr{P}]_{H} \Subset H$, является равномерно (по $g \in$ $\left.\mathscr{H}\left(g_{0}\right)\right)$ притягиваюшим множеством семейства процессов $\left\{U_{g}(t, \tau), g \in \mathscr{H}\left(g_{0}\right)\right\}$. Как и выше, предполагается, что $\left\{U_{g}(t, \tau)\right\} u_{\tau}$ непрерывно по $\left(u_{\tau}, g\right) \in E \times \mathscr{H}\left(g_{0}\right)$, принимая значения в $E$. Тогда для равномерного аттрактора $\mathscr{A}$ семейства процессов $\left\{U_{g}(t, \tau), g \in \mathscr{H}\left(g_{0}\right)\right\}$ имеет место формула

$$
\mathscr{A}=\bigcap_{h \geqslant 0}\left[\bigcup_{t-\tau \geqslant h} U_{g_{0}}(t, \tau) \mathscr{P}\right]_{E} .
$$

§2. Диссипативное гиперболическое уравнение с быстро осциллирующей по $t$ внешней силой и его глобальный аттрактор

Рассматривается диссипативное гиперболическое уравнение вида

$$
\partial_{t}^{2} u+\gamma \partial_{t} u=\Delta u-\delta^{2} u-f(u)+g\left(x, \frac{t}{\varepsilon}\right), \quad x \in \mathbb{T}^{n},
$$

где $\gamma>0, \delta^{2}>0,0<\varepsilon \leqslant \varepsilon_{0}$, при периодических граничных условиях: $x \in \mathbb{T}^{n}$. Как известно, уравнению (2.1) можно придать вид (1.9) эволюционного уравнения:

$$
\left\{\begin{array}{l}
\partial_{t} u=p \\
\partial_{t} p=-\gamma p+\Delta u-\delta^{2} u-f(u)+g\left(x, \frac{t}{\varepsilon}\right)
\end{array}\right.
$$

Обозначив $(u, p)=y$, эту систему можно записать в виде (1.9):

$$
\partial_{t} y=L(y)+G\left(x, \frac{t}{\varepsilon}\right), \quad G=(0, g), \quad x \in \mathbb{T}^{n} .
$$

Однако ниже в основном используется уравнение (2.1). Предполагается, что функция $g(x, z), z \in \mathbb{R}, x \in \mathbb{T}^{n},-$ тр.к. по $z$ в $L_{2}^{\operatorname{loc}}(\mathbb{R}, H)\left(H=L_{2}\left(\mathbb{T}^{n}\right)\right)$, т.е.

$$
\begin{gathered}
\{g(\cdot, z+h), h \in \mathbb{R}\}-\text { предкомпактное множество в } L_{2}\left(\left[z_{1}, z_{2}\right], H\right) \\
\text { при любых } z_{1}, z_{2} \in \mathbb{R}, z_{1}<z_{2} .
\end{gathered}
$$


Отсюда следует, что

$$
\|g(\cdot, \cdot)\|_{L_{2}^{b}}^{2}:=\sup _{z \in \mathbb{R}} \int_{z}^{z+1}|g(\cdot, \xi)|^{2} d \xi \leqslant \frac{1}{2} M<+\infty,
$$

здесь и далее $|\cdot|=\|\cdot\|_{H}($ см. [1], [11]). Кроме того,

$$
\begin{aligned}
\left\|g\left(\cdot, \frac{\dot{-}}{\varepsilon}\right)\right\|_{L_{2}^{b}} & :=\sup _{t \in \mathbb{R}} \int_{t}^{t+1}\left|g\left(\cdot, \frac{\tau}{\varepsilon}\right)\right|^{2} d \tau \\
& =\varepsilon \sup _{t \in \mathbb{R}} \int_{t / \varepsilon}^{(t+1) / \varepsilon}|g(\cdot, z)|^{2} d z \leqslant M, \quad 0<\varepsilon \leqslant \varepsilon_{0} .
\end{aligned}
$$

Относительно функции взаимодействия $f(v)$ предполагается, что

$$
\begin{gathered}
f(v) \in C^{1}(\mathbb{R}), \quad\left|f^{\prime}(v)\right| \leqslant C_{0}\left(|v|^{\rho}+1\right), \\
0 \leqslant \rho<\frac{2}{n-2}, \quad n \geqslant 3 ; \quad n=1,2, \quad \rho-\text { любое число, } \quad \rho \in \mathbb{R} .
\end{gathered}
$$

Кроме того,

$$
\begin{gathered}
F(v) \geqslant-m v^{2}-C_{m}, \quad F(v)=\int_{0}^{v} f(w) d w, \\
f(v) v-\gamma_{1} F(v)+m v^{2} \geqslant-C_{m},
\end{gathered}
$$

где $m>0, \gamma_{1}>0, m$ достаточно мало.

Предполагается, что внешняя сила $g(x, t / \varepsilon)$ обладает равномерным усреднением $\bar{g}(x)$ в $L_{2, w}^{\text {loc }}(\mathbb{R}, H)$ при $\varepsilon \searrow 0$. Это означает, что для любой функции $\varphi(x, t)$, принадлежашей $L_{2}([-T, T], H)$, где $T>0$ - любое число из $\mathbb{R}_{+}$,

$$
\int_{-T}^{+T}\left\langle g\left(\cdot, \frac{t+h}{\varepsilon}\right), \varphi(\cdot, t)\right\rangle_{H} d t \underset{\varepsilon \rightarrow 0+}{\longrightarrow} \int_{-T}^{+T}\langle\bar{g}(\cdot), \varphi(\cdot, t)\rangle_{H} d t
$$

равномерно по $h \in \mathbb{R}$ (см. [9]). Сформулируем достаточное условие, при котором (2.7) вьполняется:

$$
\frac{1}{\lambda} \int_{h}^{h+\lambda} g\left(x, z_{1}\right) d z_{1} \underset{\lambda \rightarrow+\infty}{\longrightarrow} \bar{g}(x) \text { в } H
$$

причем эта сходимость равномерная по $h \in \mathbb{R}$.

Отметим, что условие (2.8) выполняется для любой п.п. по $z$ функции $g(x, z)$ со значениями в $H$ (см. [12]).

Пусть $\mathscr{H}(g(x, z))$ - оболочка функции $g(x, z)$ в $L_{2}^{\operatorname{loc}}(\mathbb{R}, H)$ и $\mathscr{H}(g(x, t / \varepsilon))$ - оболочка функции $g(x, t / \varepsilon)$ в $L_{2}^{\text {loc }}(\mathbb{R}, H)$ (при фиксированном $\varepsilon, 0<\varepsilon \leqslant \varepsilon_{0}$ ). Так как $g(x, z)$ - тр.к. в $L_{2}^{\text {loc }}(\mathbb{R}, H)($ см. $(2.3))$, то $\widehat{g}(x, t / \varepsilon) \in \mathscr{H}(g(x, t / \varepsilon))$, если существует последовательность $\left\{h_{n}\right\}, h_{n} \in \mathbb{R}$, такая, что

$$
g\left(\cdot, \frac{t+h_{n}}{\varepsilon}\right) \underset{n \rightarrow+\infty}{\longrightarrow} \widehat{g}\left(\cdot, \frac{t}{\varepsilon}\right) \quad \text { в } \quad L_{2}^{\mathrm{loc}}([-T, T], H) \quad \forall T>0
$$


Обозначим для краткости $\widehat{g}(x, t / \varepsilon):=\widehat{g}^{\varepsilon}(t):=\widehat{g}(t), g(x, t / \varepsilon):=g^{\varepsilon}(t):=g(t)$. Имеют место равномерные оценки

$$
\left\|\widehat{g}^{\varepsilon}\right\|_{L_{2}^{b}}^{2}=\sup _{t \in \mathbb{R}} \int_{t}^{t+1}\left|\widehat{g}^{\varepsilon}\left(\cdot, t_{1}\right)\right|^{2} d t_{1} \leqslant\left\|g^{\varepsilon}\right\|_{L_{2}^{b}} \leqslant M
$$

где $M$ - такое же, как в $\left(2.4^{\prime}\right)$. Из (2.7) следует, что функция $\widehat{g}(x, t / \varepsilon)$ обладает при $\varepsilon \rightarrow 0+$ таким же равномерньм усреднением $\bar{g}(x)$ в $L_{2, w}^{\text {loc }}(\mathbb{R}, H)$, как и функция $g(x, t / \varepsilon)$ :

$$
\widehat{g}\left(x, \frac{t}{\varepsilon}\right) \underset{\varepsilon \rightarrow 0+}{\longrightarrow} \bar{g}(x) \quad \text { в } \quad L_{2, w}^{\text {loc }}(\mathbb{R}, H)
$$

(см. [9]).

Кроме того, предполагается, что функция $g(x, z)$ тр.к. по $z$ в $L_{2}^{\text {loc }}\left(\mathbb{R}, H_{\sigma_{2}}\right)$, где $\sigma_{2}=1-\rho(n-2) / 2, n \geqslant 3, \rho$ - такое же, как в $(2.5)$. Отсюда следует, что $\partial_{x_{i}} g(x, z)$, $i=1, \ldots, n$, тр.к. по $z$ в $L_{2}^{\text {loc }}\left(\mathbb{R}, H_{\sigma_{2}-1}\right)$. Следовательно, для $\widehat{g} \in \mathscr{H}(g)$ имеем

$$
\begin{gathered}
\sup _{t \in \mathbb{R}} \int_{t}^{t+1}\left\|\widehat{g}_{x_{i}}\left(\cdot, \frac{t_{1}}{\varepsilon}\right)\right\|_{H_{\sigma_{2}-1}}^{2} d t_{1} \leqslant M_{1}<+\infty \\
\forall \widehat{g} \in \mathscr{H}(g), \quad i=1, \ldots, n, \quad 0<\varepsilon \leqslant \varepsilon_{0} .
\end{gathered}
$$

Отметим, что $M_{1}$ не зависит от $\varepsilon$ (см. $\left.(2.4),\left(2.4^{\prime}\right)\right)$ и от $\widehat{g} \in \mathscr{H}(g)$.

Зададим при $t=\tau$ начальные условия:

$$
\left.u\right|_{t=\tau}=u_{0}(x),\left.\quad \partial_{t} u\right|_{t=\tau}=u_{1}(x), \quad\left(u_{0}, u_{1}\right):=y(\tau) \in H_{1} \times H:=E .
$$

Наряду с уравнением (2.1) рассмотрим усредненное уравнение

$$
\partial_{t}^{2} \bar{u}+\gamma \partial_{t} \bar{u}=\Delta \bar{u}-\delta^{2} \bar{u}-f(\bar{u})+\bar{g}(x), \quad x \in \mathbb{T}^{n},
$$

где $\bar{g}(x)$ - усреднение функции $g(x, t / \varepsilon)$ в $L_{2, w}^{\text {loc }}(\mathbb{R}, H)$ при $\varepsilon \rightarrow 0+$. Задача $(2.1)$, $(2.12)$ и задача $(2.13),(2.12)$ имеют, и притом единственное, решение

$$
\begin{gathered}
y_{g}(t):=y(t)=\left(u(t), \partial_{t} u(t)\right)=U_{g}(t, \tau) y(\tau), \quad y(\tau)=\left(u_{0}, u_{1}\right), \\
y_{\bar{g}}(t):=\left(\bar{u}(t), \partial_{t} \bar{u}(t)\right)=U_{\bar{g}}(t, \tau) y(\tau) .
\end{gathered}
$$

Так как уравнение (2.13) автономно, то $U_{\bar{g}}(t, \tau) y(\tau)=S(t-\tau) y(\tau)$, где $\left\{S\left(t_{1}\right)\right.$, $\left.t_{1} \geqslant 0\right\}-$ полугруппа, порожденная (2.13). Доказательство этих фактов стандартно (см., например, [2], [4]). Аналогично, если в (2.1) $g=\widehat{g}, \widehat{g} \in \mathscr{H}(g)$, то соответствуюшая задача Коши (2.1), (2.12) имеет, и притом единственное, решение $y_{\widehat{g}}(t)=U_{\widehat{g}}(t, \tau) y_{\widehat{g}}(\tau)$.

Ниже изучается семейство процессов $\left\{U_{\widehat{g}}(t, \tau), \widehat{g} \in \mathscr{H}(g)\right\}$, порожденное уравнениями вида (2.1), где $g$ заменено на $\widehat{g}$. Сначала устанавливается сушествование равномерно притягиваюшего множества $\mathscr{P}$ для этого семейства процессов.

ТЕОРемА 2.1. При выполнении указанных выше условий при любом фиксированном $\varepsilon, 0<\varepsilon \leqslant \varepsilon_{0}$, семейство прочессов

$$
\left\{U_{\widehat{g}}(t, \tau), \widehat{g}\left(x, \frac{t}{\varepsilon}\right):=\widehat{g} \in \mathscr{H}\left(g\left(x, \frac{t}{\varepsilon}\right)\right)\right\} \cup\left\{U_{\bar{g}}(t, \tau)\right\}
$$

обладает равномерно (по $\widehat{g}$ и по $\bar{g}$ ) притягивающим множеством $\mathscr{P}$, компактным в Е. Множество Р્ не зависит от $\varepsilon, 0<\varepsilon \leqslant \varepsilon_{0}$. 
ДокАЗАтельство. Рассматривается задача Коши

$$
\begin{gathered}
\partial_{t}^{2} \widehat{u}+\gamma \partial_{t} \widehat{u}=\Delta \widehat{u}-\gamma^{2} \widehat{u}-f(\widehat{u})+\widehat{g}\left(x, \frac{t}{\varepsilon}\right), \quad x \in \mathbb{T}^{n}, \\
\left.\widehat{u}\right|_{t=\tau}=u_{0}(x),\left.\quad \partial_{t} \widehat{u}\right|_{t=\tau}=u_{1}(x), \quad\left(u_{0}(x), u_{1}(x)\right):=y_{\tau}(x) \in E .
\end{gathered}
$$

Здесь $\widehat{g}(x, t / \varepsilon) \in \mathscr{H}(g(x, t / \varepsilon))$. Решение $\widehat{u}_{\widehat{g}}(x, t)$ задачи $(2.15),(2.16)$ можно представить в виде

$$
y_{\widehat{g}}(t):=\widehat{y}=\Sigma(t-\tau) y_{\tau}+\widetilde{U}_{\widehat{g}}(t, \tau) y_{\tau}
$$

Здесь

$$
\Sigma(t-\tau) y_{\tau}=\left(u_{2}(t), \partial_{t} u_{2}(t)\right):=y_{2}(t),
$$

где $u_{2}(t)$ - решение следуюшей линейной задачи:

$$
\begin{aligned}
\partial_{t}^{2} u_{2}+\gamma \partial_{t} u_{2}+A u_{2} & =0, \quad A v(x):=-\Delta v(x)+\delta^{2} v(x), \quad x \in \mathbb{T}^{n}, \\
\left.u_{2}\right|_{t=\tau} & =u_{0}(x),\left.\quad \partial_{t} u_{2}\right|_{t=\tau}=u_{1}(x) .
\end{aligned}
$$

Как известно (см. [1], [2]), решение $\left(u_{2}(t), \partial_{t} u_{2}(t)\right):=y_{2}(t)$ задачи $(2.19),(2.20)$ экспоненциально убывает по $(t-\tau)$ :

$$
\left\|y_{2}(t)\right\|_{E} \leqslant\left\|y_{\tau}\right\|_{E} e^{-\alpha_{1}(t-\tau)}, \quad \alpha_{1}>0, \quad t \geqslant \tau
$$

Определим второе слагаемое в (2.17):

$$
\begin{gathered}
\widetilde{y}(t):=\left(\widetilde{u}(t), \partial_{t} \widetilde{u}(t)\right):=\widetilde{U}_{\widehat{g}}(t, \tau) y_{\tau}, \\
y_{\tau}=\left(u_{0}(x), u_{1}(x)\right)=\left(\left.\widehat{u}\right|_{t=\tau},\left.\partial_{t} \widehat{u}\right|_{t=\tau}\right),
\end{gathered}
$$

причем функция $\widetilde{u}(t)$ удовлетворяет уравнению

$$
\begin{aligned}
& \partial_{t}^{2} \widetilde{u}+\gamma \partial_{t} \widetilde{u}+A \widetilde{u}=\widehat{g}\left(x, \frac{t}{\varepsilon}\right)-f(\widehat{u}(t)), \quad x \in \mathbb{T}^{n}, \\
& \left.\left.\widetilde{u}\right|_{t=\tau}=0,\left.\quad \partial_{t} \widetilde{u}\right|_{t=\tau}=0 \quad \text { (или }\left.\widetilde{y}\right|_{t=\tau}=\widetilde{y}_{\tau}=0\right) .
\end{aligned}
$$

Отметим, что $\widetilde{y}(t)$ однозначно определяется через $y_{\tau}=\left(u_{0}(x), u_{1}(x)\right)$, так как $\widetilde{u}(t)$ однозначно определяется через $y_{\tau}$ (см. (2.15), (2.16)). Действительно, через $\widehat{u}(t)$ выражается правая часть (2.23). Зная правую часть (2.23), мы однозначно находим $\left(\widetilde{u}(t), \partial_{t} \widetilde{u}(t)\right)=\widetilde{y}(t)-$ решение задачи Коши для $(2.23),(2.24)$. Заметим, что семейство операторов $\left\{\widetilde{U}_{\widehat{g}}(t, \tau)\right\}$ не является процессом.

Докажем сначала следуюшую лемму.

Лемма 2.1. Семейство процессов $\left\{U_{\widehat{g}}(t, \tau), \widehat{g}=\widehat{g}(x, t / \varepsilon) \in \mathscr{H}(g(x, t / \varepsilon))\right\}$, $0<\varepsilon \leqslant \varepsilon_{0}$, обладает равномерным (по $\widehat{g}$ ) поглощающим множеством $B_{0}$, ограниченным в $E$. Множество $B_{0}$ не зависит от $\varepsilon$. 
Доказательство. Решение задачи $(2.15),(2.16)\left(\widehat{u}(t), \partial_{t} \widehat{u}(t)\right):=\widehat{y}(t) \in$ $C_{b}\left(\mathbb{R}_{\tau}, E\right)($ см. [1]) удовлетворяет следующей оценке:

$$
\begin{gathered}
\|\widehat{y}(t)\|_{E}^{2}:=\|\widehat{u}(t)\|_{H_{1}}^{2}+\left\|\partial_{t} \widehat{u}(t)\right\|_{H}^{2}:=\|\widehat{u}(t)\|_{1}^{2}+\left|\partial_{t} \widehat{u}(t)\right|^{2} \\
\leqslant C_{4}\left(\|y(\tau)\|_{E}^{\rho+2}+1\right) e^{-\beta(t-\tau)}+\int_{\tau}^{t}\left(C^{\prime}+C_{1}^{\prime}\left|\widehat{g}\left(\tau_{1}\right)\right|_{H}^{2}\right) e^{-\beta\left(t-\tau_{1}\right)} d \tau_{1}, \\
H=H\left(\mathbb{T}^{n}\right), \quad H_{1}=H_{1}\left(\mathbb{T}^{n}\right), \quad \beta>0 .
\end{gathered}
$$

Здесь $C_{4}, C^{\prime}, C_{1}^{\prime}$ - константы, не зависяшие от $\widehat{y}(\tau)=y_{\tau} \in E, \widehat{g} \in \mathscr{H}(g)$. Ниже приводится идея доказательства (2.25).

Сначала заметим, что последний интеграл в (2.25) допускает следуюшую оценкy:

$$
\begin{aligned}
& \int_{\tau}^{t}\left(C^{\prime}+C_{1}^{\prime}\left|\widehat{g}\left(\tau_{1}\right)\right|^{2}\right) e^{-\beta\left(t-\tau_{1}\right)} d \tau_{1} \\
& \leqslant C_{2}^{\prime}+C_{3}^{\prime}\left(\int_{t-1}^{t}\left|\widehat{g}\left(\tau_{1}\right)\right|^{2} e^{-\beta\left(t-\tau_{1}\right)} d \tau_{1}+\int_{t-2}^{t-1}\left|\widehat{g}\left(\tau_{1}\right)\right|^{2} e^{-\beta\left(t-\tau_{1}\right)} d \tau_{1}+\cdots\right) \\
& \leqslant C_{2}^{\prime}+C_{3}^{\prime}\left(\int_{t-1}^{t}\left|\widehat{g}\left(\tau_{1}\right)\right|^{2} d \tau_{1}+e^{-\beta} \int_{t-2}^{t-1}\left|\widehat{g}\left(\tau_{1}\right)\right|^{2} d \tau_{1}+e^{-2 \beta} \int_{t-3}^{t-2}\left|\widehat{g}\left(\tau_{1}\right)\right|^{2} d \tau_{1}+\cdots\right) \\
& \leqslant C_{2}^{\prime}+C_{3}^{\prime}\left(1-e^{-\beta}\right)^{-1}\|\widehat{g}\|_{L_{2}^{b}}^{2} \leqslant C_{3}(M), \\
& \text { где }\|\widehat{g}\|_{L_{2}^{b}}^{2} \leqslant M(\text { см. (2.10)). }
\end{aligned}
$$

Отсюда и из (2.25) следует, что

$$
\|\widehat{y}(t)\|_{E}^{2} \leqslant C_{4}\left(\|\widehat{y}(\tau)\|_{E}^{\rho+2}+1\right) e^{-\beta(t-\tau)}+C_{3}(M) .
$$

Доказательство оценки (2.25) дано в [1; гл. VI, лемма 4.1]. Приведем лишш основную идею этого доказательства. Введем функцию

$$
z(t)=\int_{\mathbb{T}^{n}}\left(|\nabla \widehat{u}(x, t)|^{2}+\delta^{2}|\widehat{u}(x, t)|^{2}+\left|\partial_{t} \widehat{u}(x, t)+\alpha \widehat{u}(x, t)\right|^{2}-2 F(\widehat{u}(x, t))\right) d x,
$$

где $\alpha>0$ и достаточно мало, $\widehat{u}(x, t)$ - решение задачи $(2.15),(2.16), F(u)=$ $\int_{0}^{u} f(v) d v$. Используя условия (2.3)-(2.6), из (2.27) выводится следуюшее дифференциальное неравенство:

$$
d_{t} z(t)+\beta z(t) \leqslant C+C_{1}|\widehat{g}(t)|^{2}, \quad \beta>0 .
$$

Отсюда, по неравенству Гронуола следует, что

$$
z(t) \leqslant z(\tau) e^{-\beta(t-\tau)}+\int_{\tau}^{t}\left(C+C_{1}\left|\widehat{g}\left(\tau_{1}\right)\right|^{2}\right) e^{-\beta\left(t-\tau_{1}\right)} d \tau_{1} .
$$

Учитьвая $\left(2.25^{\prime}\right)$ и степенной рост функции $F(u)$, из $(2.28)$ выводится $(2.25)$ (см. [1; гл. VI, лемма 4.1]). 
Из неравенства (2.26) следует, что множество

$$
B_{0}=\left\{y \subset E \mid\|y\|_{E}^{2} \leqslant 2 C_{3}(M)\right\}
$$

является равномерно (по $\widehat{g}=\widehat{g}(x, t / \varepsilon) \in \mathscr{H}(g), \tau \in \mathbb{R}$ и $\varepsilon, 0<\varepsilon \leqslant \varepsilon_{0}$ ) поглощающим множеством для семейства процессов $\left\{U_{\widehat{g}}(t, \tau), t \geqslant \tau, \tau \in \mathbb{R}, \widehat{g} \in \mathscr{H}(g)\right\}$ при любом фиксированном $\varepsilon$.

Заметим, что $B_{0}$ не зависит от $\varepsilon, 0<\varepsilon \leqslant \varepsilon_{0}$, так как $C_{3}=C_{3}(M)$, а $M$ не зависит от $\varepsilon$ (см. (2.4), $\left.\left(2.4^{\prime}\right)\right)$.

Кроме того, из (2.26) следует, что для любого ограниченного множества $B_{1} \in$ $\mathscr{B}(E)$

$$
U_{\widehat{g}}(\tau+h, \tau) B_{1} \subset B_{0}, \quad t \geqslant h\left(B_{1}\right),
$$

где $h\left(B_{1}\right)$ не зависит от $\widehat{g} \in \mathscr{H}(g)$, а также от $\tau \in \mathbb{R}$ и от $\varepsilon, 0<\varepsilon \leqslant \varepsilon_{0}$. Таким образом, без ограничения общности (как будет доказано ниже) можно считать, что $y_{\tau}=\widehat{y}(\tau) \in B_{0}$.

Продолжим теперь доказательство теоремы 2.1.

Применим оценку (2.25) к уравнению $(2.23)$, считая, что член $\widehat{g}(x, t / \varepsilon)+f(\widehat{u}(t))$ является известной правой частью, а начальное условие $\left.\widetilde{y}\right|_{t=\tau}=\left(\widetilde{u}(\tau), \partial_{t} \widetilde{u}(\tau)\right)=0$ (согласно (2.24)). Учитывая (2.23), (2.24) и тот факт, что в левой части (2.23) отсутствует нелинейный член, мы получаем

$$
\begin{aligned}
\|\widetilde{y}(t)\|_{E}^{2} & :=\|\widetilde{u}(t)\|_{1}^{2}+\left|\partial_{t} \widetilde{u}(t)\right|^{2} \\
& \leqslant C \int_{\tau}^{t}\left(\left|\widehat{g}\left(t_{1}\right)\right|^{2}+\left|f\left(\widehat{u}\left(t_{1}\right)\right)\right|^{2}\right) e^{-\alpha_{1}\left(t-t_{1}\right)} d t_{1} \\
& \leqslant C_{5}+C_{6}\|\widehat{g}\|_{L_{2}^{b}}^{2}+C_{7} \int_{\tau}^{t}\left(\left\|\widehat{y}\left(t_{1}\right)\right\|_{E}^{2(\rho+1)}+1\right) e^{-\alpha_{1}\left(t-t_{1}\right)} d t_{1} \\
& \leqslant C\left(M,\left\|B_{0}\right\|_{E}\right), \quad \alpha_{1}>0
\end{aligned}
$$

(см. [1], [2]). При этом мы воспользовались тем, что $\widehat{y}(\tau) \in B_{0}$, оценками $(2.25)$, $\left(2.25^{\prime}\right)$ и тем, что $\|\widehat{g}\|_{L_{2}^{b}}^{2} \leqslant M$. Отметим, что оценка $(2.31)$ не зависит от $\varepsilon$, $0<\varepsilon \leqslant \varepsilon_{0}$.

Дифференцируя (2.23) и (2.24) по $x_{i}$, получаем

$$
\begin{gathered}
\partial_{t}^{2}\left(\partial_{x_{i}} \widetilde{u}\right)+\gamma \partial_{t}\left(\partial_{x_{i}} \widetilde{u}\right)-\Delta\left(\partial_{x_{i}} \widetilde{u}\right)+\delta^{2} \partial_{x_{i}} \widetilde{u}=f_{u}^{\prime}(\widehat{u}) \partial_{x_{i}} \widehat{u}+\widehat{g}_{x_{i}}\left(x, \frac{t}{\varepsilon}\right), \\
\left.\partial_{x_{i}} \widetilde{u}\right|_{t=\tau}=0,\left.\quad \partial_{t}\left(\partial_{x_{i}} \widetilde{u}\right)\right|_{t=\tau}=0, \quad i=1, \ldots, n
\end{gathered}
$$

Обозначив в $(2.32)$ и (2.33) $\partial_{x_{i}} \widetilde{u}:=v$, получим

$$
\begin{gathered}
\partial_{t}^{2} v+\gamma \partial_{t} v+A v=-f_{u}^{\prime}(\widehat{u}) \partial_{x_{i}} \widehat{u}+\widehat{g}_{x_{i}}^{\prime}\left(x, \frac{t}{\varepsilon}\right), \\
A v=-\Delta v+\delta^{2} v \\
\left.v\right|_{t=\tau}=0,\left.\quad \partial_{t} v\right|_{t=\tau}=0 .
\end{gathered}
$$


Пусть $\sigma_{2}=1-\rho(n-2) / 2, n \geqslant 3$, где $\rho$ - такое же, как в $(2.5)$. Очевидно $\sigma_{2}>0$. (Случай $n=1,2$ трактуется аналогично.)

Для $\theta(x) \in L_{2}\left(\mathbb{T}^{n}\right):=H$ и $\nu(x) \in H_{1}\left(\mathbb{T}^{n}\right)$ имеет место неравенство

$$
\left\|f^{\prime}(\nu) \theta\right\|_{H_{\sigma_{2}-1}} \leqslant C_{2}^{\prime} C_{3}^{\prime}\left(\|\nu\|_{1}\right)|\theta|
$$

где $C_{3}^{\prime}(s)$ - возрастающая степенная функция (степень которой определяется с помощью теоремы вложения Соболева). Доказательство (2.36) дано в [2; гл. IV, лемма 3.3]. Применяя оператор $A^{\left(\sigma_{2}-1\right) / 2}$ к обеим частям $(2.34)$ и $(2.35)$, получаем

$$
\begin{gathered}
\partial_{t}^{2}\left(A^{\left(\sigma_{2}-1\right) / 2} v\right)+\gamma \partial_{t}\left(A^{\left(\sigma_{2}-1\right) / 2} v\right)+A\left(A^{\left(\sigma_{2}-1\right) / 2} v\right) \\
=-A^{\left(\sigma_{2}-1\right) / 2}\left(f_{u}^{\prime}(\widehat{u}) \partial_{x_{i}} \widehat{u}\right)+A^{\left(\sigma_{2}-1\right) / 2} \widehat{g}_{x_{i}}^{\prime}\left(x, \frac{t}{\varepsilon}\right), \\
\left.A^{\left(\sigma_{2}-1\right) / 2} v\right|_{t=\tau}=0,\left.\quad \partial_{t} A^{\left(\sigma_{2}-1\right) / 2} v\right|_{t=\tau}=0 .
\end{gathered}
$$

Отметим, что из (2.36) следует

$$
\begin{aligned}
\left|A^{\left(\sigma_{2}-1\right) / 2}\left(f^{\prime}(\widehat{u}(t)) \partial_{x_{i}} \widehat{u}\right)\right| & \leqslant C\left\|f^{\prime}(\widehat{u}) \partial_{x_{i}} \widehat{u}\right\|_{H_{\sigma_{2}-1}} \\
& \leqslant C C_{2}^{\prime} C_{3}^{\prime}\left(\|\widehat{u}(t)\|_{1}\right)\left|\partial_{x_{i}} \widehat{u}(t)\right| \\
& \leqslant C_{0}\left(\left\|B_{0}\right\|_{E}, M\right) .
\end{aligned}
$$

В последнем неравенстве мы воспользовались тем, что $\left(\widehat{u}(\tau), \partial_{t} \widehat{u}(\tau)\right)=\widehat{y}(\tau) \in$ $B_{0}$ и оценкой $(2.25)$, согласно которой

$$
\|\widehat{y}(t)\|_{E}^{2}=\left\|\left(\widehat{u}, \partial_{t} \widehat{u}(t)\right)\right\|_{E}^{2} \leqslant C_{4}\left(\left\|B_{0}\right\|_{E}, M\right) \quad \forall t \geqslant \tau .
$$

Уравнение $(2.37)$ относительно $\left(A^{\left(\sigma_{2}-1\right) / 2} v\right):=w$ является неоднородным линейным гиперболическим уравнением с правой частью

$$
-A^{\left(\sigma_{2}-1\right) / 2}\left(f_{u}^{\prime}(\widehat{u}(t)) \partial_{x_{i}} \widehat{u}(t)\right)+A^{\left(\sigma_{2}-1\right) / 2}\left(\widehat{g}_{x_{i}}^{\prime}\left(\frac{t}{\varepsilon}\right)\right):=\varphi(t) .
$$

Таким образом, имеем

$$
\partial_{t}^{2} w+\gamma \partial_{t} w+A w=\varphi(t),\left.\quad w\right|_{t=\tau}=0,\left.\quad \partial_{t} w\right|_{t=\tau}=0
$$

Как известно, для $w(t)$ имеет место оценка

$$
\|w(t)\|_{1}^{2}+\left|\partial_{t} w(t)\right|^{2} \leqslant C_{1} \int_{\tau}^{t}\left|\varphi\left(\tau_{1}\right)\right|^{2} e^{-\alpha_{1}\left(t-\tau_{1}\right)} d \tau_{1},
$$

где $\alpha_{1}>0$.

Отсюда, подставляя вместо $w(t)$ и $\varphi(t)$ их выражения, получаем

$$
\begin{aligned}
\|v\|_{H_{\sigma_{2}}}^{2}+\left\|\partial_{t} v\right\|_{H_{\sigma_{2}-1}}^{2} \leqslant & C_{2}\left(\left\|A^{\left(\sigma_{2}-1\right) / 2} v(t)\right\|_{1}^{2}+\left|\partial_{t} A^{\left(\sigma_{2}-1\right) / 2} v(t)\right|^{2}\right) \\
\leqslant & C_{3} \int_{\tau}^{t}\left(\left|A^{\left(\sigma_{2}-1\right) / 2}\left(f^{\prime}\left(\widehat{u}\left(\tau_{1}\right)\right) \partial_{x_{i}} \widehat{u}\left(\tau_{1}\right)\right)\right|^{2}\right. \\
& \left.+\left|A^{\left(\sigma_{2}-1\right) / 2} \widehat{g}_{x_{i}}^{\prime}\left(\cdot, \frac{\tau_{1}}{\varepsilon}\right)\right|^{2}\right) e^{-\alpha_{1}\left(t-\tau_{1}\right)} d \tau_{1}
\end{aligned}
$$


Учитьвая оценки (2.39) и (2.11), из (2.40) выводим

$$
\begin{aligned}
\|v\|_{H_{\sigma_{2}}}^{2}+\left\|\partial_{t} v\right\|_{H_{\sigma_{2}-1}}^{2} & \leqslant C_{5}\left(\left\|B_{0}\right\|_{E}, M\right)+C_{6}\left(\left\|\widehat{g}_{x_{i}}\right\|_{L_{2}^{b}\left(H_{\sigma_{2}-1}\right)}\right) \\
& \leqslant C_{7}\left(\left\|B_{0}\right\|_{E}, M, M_{1}\right)
\end{aligned}
$$

где $M_{1}$ выражается по формуле (2.11). Отметим, что при оценке последнего слагаемого в (2.40) мы использовали $\left(2.25^{\prime}\right)$. Так как $v=\partial_{x_{i}} \widetilde{u}, i=1, \ldots, n$, то из $(2.41)$ следует

$$
\sum_{i=1}^{n}\left(\left\|\partial_{x_{i}} \widetilde{u}(t)\right\|_{H_{\sigma_{2}}}+\left\|\partial_{t}\left(\partial_{x_{i}} \widetilde{u}(t)\right)\right\|_{H_{\sigma_{2}-1}}\right) \leqslant C_{8}\left(\left\|B_{0}\right\|_{E}, M, M_{1}\right), \quad t \geqslant \tau .
$$

Согласно оценке (2.31)

$$
\|\widetilde{y}(t)\|_{E}^{2}=\|\widetilde{u}(t)\|_{1}^{2}+\left|\partial_{t} \widetilde{u}(t)\right|^{2} \leqslant C\left(\left\|B_{0}\right\|_{E}, M\right) .
$$

Отсюда и из (2.42) следует

$$
\begin{aligned}
\|\widetilde{y}(t)\|_{E_{\sigma_{2}}} & :=\left(\|\widetilde{u}(t)\|_{H_{\sigma_{2}+1}}^{2}+\left\|\partial_{t} u(t)\right\|_{H_{\sigma_{2}}}^{2}\right)^{1 / 2} \\
& \leqslant C_{9}\left(\left\|B_{0}\right\|_{E}, M, M_{1}\right), \quad t \geqslant \tau, \quad \tau \in \mathbb{R}, \quad \sigma_{2}>0 .
\end{aligned}
$$

Так как $\sigma_{2}>0$, то в силу (2.43) множество $\mathscr{P}=\bigcup_{0<\varepsilon \leqslant \varepsilon_{0}}\{\widetilde{y}(t), t \geqslant \tau$, $\tau \in \mathbb{R} \mid \widetilde{y}(t)$ - решение задачи Коши (2.23), (2.24), $\widehat{g} \in \mathscr{H}(g)$ и $\widehat{u}$ - решение задачи Коши $(2.15),(2.16)$ с $\left.y_{\tau}=\left(u_{0}(x), u_{1}(x)\right) \in B_{0}\right\}$ предкомпактно в $E$. Покажем теперь, что $\mathscr{P}$ является равномерно притягивающим множеством для семейства процессов $\left\{U_{\widehat{g}}(t, \tau), \widehat{g} \in \mathscr{H}(g), \widehat{g}=\widehat{g}(x, t / \varepsilon)\right\}$ и притом в силу (2.43) $\mathscr{P}$ предкомпактно в $E$.

Действительно, пусть $B_{1} \in \mathscr{B}(E)$ - любое ограниченное множество в $E$.

Так как $B_{0}$ - равномерно поглощающее множество для семейства процессов $\left\{U_{\widehat{g}}(t, \tau), \widehat{g} \in \mathscr{H}(g), \widehat{g}=\widehat{g}(x, t / \varepsilon)\right\}$, то найдется такое $h=h\left(B_{1}\right)$, что $\forall \tau_{1} \geqslant \tau+$ $h\left(B_{1}\right):=\tau_{0}\left(\tau, B_{1}\right):=\tau_{0}$

$$
U_{\widehat{g}}\left(t_{1}, \tau\right) B_{1} \subset B_{0}
$$

Поэтому при $t \geqslant \tau_{0}$ в силу $(2.17)$

$$
U_{\widehat{g}}\left(t, \tau_{0}\right) B_{0}=\Sigma\left(t-\tau_{0}\right) B_{0}+\widetilde{U}\left(t, \tau_{0}\right) B_{0} \subset \Sigma\left(t-\tau_{0}\right) B_{0}+\mathscr{P} .
$$

Так как $\Sigma\left(t-\tau_{0}\right)$ экспоненциально убывает, то для любого $\eta>0$

$$
U_{\widehat{g}}\left(t, \tau_{0}\right) B_{0} \subset \mathscr{O}_{\eta}(\mathscr{P}), \quad t-\tau_{0} \geqslant \tau_{1}(\eta) .
$$

Следовательно,

$U_{\widehat{g}}(t, \tau) B_{1} \subset U_{\widehat{g}}\left(t, \tau_{0}\right) U\left(\tau_{0}, \tau\right) B_{1} \subset U_{\widehat{g}}\left(t, \tau_{0}\right) B_{0} \subset \mathscr{O}_{\eta}(\mathscr{P}), \quad t>\tau+h\left(B_{1}\right)+\tau_{1}(\eta)$

Таким образом, $\mathscr{P}$ является равномерно по $\widehat{g}^{\varepsilon}=\widehat{g}(x, t / \varepsilon) \in \mathscr{H}(g(x, t / \varepsilon)):=$ $\mathscr{H}\left(g^{\varepsilon}\right)$ притягивающим множеством процессов $\left\{U_{\widehat{g}} \varepsilon(t, \tau)\right\}$ при фиксированном $\varepsilon$, $0<\varepsilon \leqslant \varepsilon_{0}$. При этом $\mathscr{P}$ не зависит от $\varepsilon$. Очевидно, что аналогичный факт имеет место и для $U_{\bar{g}}(t, \tau)=S(t-\tau)$.

Для доказательства сушествования равномерного (по $\left.\widehat{g}^{\varepsilon} \in \mathscr{H}\left(g^{\varepsilon}\right)\right)$ аттрактора $\mathscr{A}_{\varepsilon}$ семейства процессов $\left\{U_{\widehat{g}} \varepsilon(t, \tau)\right\}$ необходимо еще установить справедливость следующего предложения (см. [1; p. 127-128]). 
ПРЕДЛОЖЕНИЕ 2.1. Семейство процессов $\left\{U_{\widehat{g}^{\varepsilon}}(t, \tau) y(\tau)\right\}$ при любом фиксированном $\varepsilon, 0<\varepsilon \leqslant \varepsilon_{0}$, является $\left(\left(E, \mathscr{H}\left(g^{\varepsilon}\right)\right), E\right)$ непрерывным (т.е. если $\left(y_{n}(\tau), \widehat{g}_{n}^{\varepsilon}\right) \rightarrow\left(y(\tau), \widehat{g}^{\varepsilon}\right)$ в $\left(E, \mathscr{H}\left(g^{\varepsilon}\right)\right)$, mo $\left.U_{\widehat{g}_{n}^{\varepsilon}}(t, \tau) y_{n}(\tau) \rightarrow U_{\widehat{g}^{\varepsilon}}(t, \tau) y(\tau)\right)$ в $E$.

Доказательство этого предложения проводится стандартно. Оно приведено в [1; c. 127-128].

Из теоремы 2.1 и предложения 2.1 следует, что семейство процессов $\left\{U_{\widehat{g}^{\varepsilon}}(t, \tau)\right\}$ обладает компактным в $E$ равномерным (по $\widehat{g}^{\varepsilon} \in \mathscr{H}^{\varepsilon}\left(g^{\varepsilon}\right)$ глобальньм аттрактором $\mathscr{A}_{\varepsilon}$.

Для $\mathscr{A}_{\varepsilon}$ имеет место следуюшая формула, аналогичная (1.16):

$$
\mathscr{A}_{\varepsilon}=\bigcap_{h \geqslant 0}\left[\bigcup_{t-\tau \geqslant h} U_{g^{\varepsilon}}(t, \tau) \mathscr{P}\right]_{E} .
$$

Отсюда и из указанных выше свойств равномерного притягиваюшего множества $\mathscr{P},[\mathscr{P}] \Subset E$, следует, что

$$
\mathscr{A}_{\varepsilon} \subset[\mathscr{P}]
$$

причем $[\mathscr{P}]$ - фиксированное, компактное в $E$ множество.

Кроме того,

$$
\mathscr{A}_{\varepsilon}=\bigcup_{\widehat{g}^{\varepsilon} \subset \mathscr{H}\left(g^{\varepsilon}\right)} \mathscr{K}_{\widehat{g}^{\varepsilon}}(0)=\bigcup_{\widehat{g}^{\varepsilon} \in \mathscr{H}\left(g^{\varepsilon}\right)} \mathscr{K}_{\widehat{g}_{\varepsilon}}(t)
$$

для любого фиксированного $t \in \mathbb{R}$. Здесь $\mathscr{K}_{\widehat{g}} \varepsilon-$ ядро процесса $\left\{U_{\widehat{g}} \varepsilon(t, \tau)\right\}:$

$$
\begin{aligned}
\mathscr{K}_{\widehat{g}^{\varepsilon}}=\left\{u^{\varepsilon}(t), t \in \mathbb{R} \mid u^{\varepsilon}(t)-\text { решение }(2.1) \text { при } t \in \mathbb{R},\right. \\
\left.\left\|\left(u^{\varepsilon}(t), \partial_{t} u^{\varepsilon}(t)\right)\right\|_{E} \leqslant M_{u^{\varepsilon}} \quad \forall t \in \mathbb{R}\right\} .
\end{aligned}
$$

\section{§ 3. Оценка отклонения траекторий}

Пусть $u^{\varepsilon}(x, t):=u_{\widehat{g}} \varepsilon(x, t):=u_{\widehat{g}}(x, t), \widehat{g}^{\varepsilon}=\widehat{g}(x, t / \varepsilon) \in \mathscr{H}\left(g^{\varepsilon}\right)=\mathscr{H}(g(x, t / \varepsilon))$, является решением уравнения

$$
\partial_{t}^{2} u^{\varepsilon}+\gamma \partial_{t} u^{\varepsilon}=\Delta u^{\varepsilon}-\delta^{2} u^{\varepsilon}-f\left(u^{\varepsilon}\right)+\widehat{g}\left(x, \frac{t}{\varepsilon}\right),
$$

$x \in \mathbb{T}^{n}$, при начальных условиях

$$
\left.u^{\varepsilon}\right|_{t=\tau}=u_{0}(x),\left.\quad \partial_{t} u^{\varepsilon}\right|_{t=\tau}=u_{1}(x),
$$

$\left(u_{0}, u_{1}\right):=y \in E$. Предполагается, что $\widehat{g}^{\varepsilon}$ и $f(v)$ обладают свойствами, сформулированными в $\S 2$. Пусть $\bar{u}(x, t):=u^{0}(x, t):=u^{0}$ - решение усредненного уравнения (2.13) с внешней силой $g^{0}(x)=\bar{g}(x)$, удовлетворяющее тем же начальным условиям, что и $u^{\varepsilon}$ :

$$
\left.u^{0}\right|_{t=\tau}=u_{0}(x),\left.\quad \partial_{t} u^{0}\right|_{t=\tau}=u_{1}(x)
$$

Предполагается, что

$$
\left\|u_{0}\right\|_{1}^{2}+\left|u_{1}\right|^{2}:=\|y\|_{E}^{2} \leqslant R^{2},
$$


где $R>0$ фиксированно. Разность $w(x, t)=u^{\varepsilon}(x, t)-u^{0}(x, t)$ удовлетворяет уравнению

$$
\partial_{t}^{2} w+\gamma \partial_{t} w=\Delta w-\delta^{2} w-\left(f\left(u^{\varepsilon}\right)-f\left(u^{0}\right)\right)+\widetilde{g}\left(x, \frac{t}{\varepsilon}\right)
$$

где $\widetilde{g}(x, t / \varepsilon)=\widehat{g}-\bar{g}=\widehat{g}-g^{0}=\widehat{g}(x, t / \varepsilon)-g^{0}(x)$, и начальным условиям

$$
\left.w\right|_{t=\tau}=0,\left.\quad \partial_{t} w\right|_{t=\tau}=0
$$

Обозначим

$$
f\left(u^{\varepsilon}\right)-f\left(u^{0}\right)=\int_{0}^{1} f_{u}^{\prime}\left(u^{0}+s\left(u^{\varepsilon}-u^{0}\right)\right) d s\left(u^{\varepsilon}-u^{0}\right):=f_{u}^{\prime} w
$$

Умножим скалярно уравнение (3.4) на $\partial_{t} w$. Учитывая (3.6), после стандартных преобразований, получаем

$$
\begin{gathered}
\frac{1}{2} \partial_{t}\left|\partial_{t} w\right|^{2}+\frac{1}{2}\left(\partial_{t}|\nabla w|^{2}+\delta^{2}|w|^{2}\right)+\gamma\left|\partial_{t} w\right|^{2}+\left\langle f_{u}^{\prime} w, \partial_{t} w\right\rangle \\
=\left\langle\widehat{g}-g^{0}, w\right\rangle:=\langle\widetilde{g}, w\rangle, \quad \widehat{g}-g^{0}:=\widetilde{g} .
\end{gathered}
$$

Предположим дополнительно, что существует первообразная $\widetilde{G}(x, z)$ функции $\widetilde{g}(x, z)$ по $z$ :

$$
\partial_{z} \widetilde{G}(x, z)=\widetilde{g}(x, z), \quad \varepsilon \partial_{t} \widetilde{G}\left(x, \frac{t}{\varepsilon}\right)=\widetilde{g}\left(x, \frac{t}{\varepsilon}\right),
$$

обладающая следующим свойством:

$$
\|\widetilde{G}(\cdot, z)\|_{1} \leqslant M_{1} \quad \forall z \in \mathbb{R}
$$

где $M_{1}$ не зависит от $\widehat{g} \in \mathscr{H}(g)$. Ниже приводятся примеры функций $\widehat{g}(x, z)-g^{0}(x)=\widetilde{g}(x)$, обладаюших свойствами (3.8), (3.9).

Интегрируя (3.7) по $t$ и используя (3.5), (3.6) и (3.8), получаем

$$
\begin{aligned}
& \frac{1}{2}\left(\left|\partial_{t} w(\tau+t)\right|^{2}+\|w(\tau+t)\|_{1}^{2}+\delta^{2}|w(\tau+t)|^{2}\right)+\gamma \int_{0}^{t}\left|\partial_{t} w(\tau+t)\right| d \tau_{1} \\
= & -\int_{0}^{t}\left\langle f_{u}^{\prime} w\left(\tau+\tau_{1}\right), \partial_{t} w\left(\tau+\tau_{1}\right)\right\rangle d \tau_{1}+\varepsilon \int_{0}^{t}\left\langle\partial_{t} \widetilde{G}\left(\frac{\tau+\tau_{1}}{\varepsilon}\right), \partial_{t} w\left(\tau+\tau_{1}\right)\right\rangle d \tau_{1} \\
= & -\int_{0}^{t}\left\langle f_{u}^{\prime} w\left(\tau+\tau_{1}\right), \partial_{t} w\left(\tau+\tau_{1}\right)\right\rangle d \tau_{1}+\varepsilon\left\langle\widetilde{G}\left(\cdot, \frac{\tau+t}{\varepsilon}\right), \partial_{t} w(\cdot, \tau+t)\right\rangle \\
& -\varepsilon \int_{0}^{t}\left\langle\widetilde{G}\left(\frac{\tau+\tau_{1}}{\varepsilon}\right), \partial_{t}^{2} w\left(\tau+\tau_{1}\right)\right\rangle d \tau_{1}:=\mathrm{I}+\mathrm{II}+\mathrm{III}
\end{aligned}
$$


Интеграл I оценим с помощью неравенства Гёльдера с показателями 1/2, $(n-2) /(2 n), 1 / n$ :

$$
\begin{aligned}
|\mathbf{I}| & =\left|\int_{0}^{t}\left\langle f_{u}^{\prime} w\left(\tau+\tau_{1}\right), \partial_{t} w\left(\tau+\tau_{1}\right)\right\rangle d \tau_{1}\right| \\
& \leqslant \int_{0}^{t}\left\|f_{u}^{\prime}\right\|_{L_{n}(\Omega)}\|w\|_{L_{2 n /(n-2)}(\Omega)}\left\|\partial_{t} w\right\|_{L_{2}(\Omega)} d \tau_{1} \\
& \leqslant C \int_{0}^{t}\left(1+\left\|u^{\varepsilon}\right\|_{\left.L_{2 n /(n-2)}^{2 /(n-2)}+\left\|u^{0}\right\|_{L_{2 n /(n-2)} /(n-2)}^{2 / 2}\right)\|w\|_{1}\left|\partial_{t} w\right| d \tau_{1}}^{t}\right. \\
& \leqslant C_{1}(R, M) \int_{0}^{t}\left(\|w\|_{1}^{2}+\left|\partial_{t} w\right|^{2}\right) d \tau_{1}, \quad\left\|y^{0}\right\|_{E}^{2},\left\|y^{\varepsilon}\right\|_{E}^{2} \leqslant C_{0}\left(R^{2}\right) .
\end{aligned}
$$

При этом мы воспользовались тем, что $\left\|\left(u_{0}^{\varepsilon}(\tau), \partial_{t} u^{\varepsilon}(\tau)\right)\right\|_{E}^{2} \leqslant R^{2}$ и, как показано в $\S 2$, отсюда следует, что $\left\|\left(u^{\varepsilon}\left(\tau+\tau_{1}\right), \partial_{t} u^{\varepsilon}\left(\tau+\tau_{1}\right)\right)\right\|_{E}^{2} \leqslant C_{0}\left(R^{2}\right)$.

Второе слагаемое справа в (3.10) оценивается с помощью (3.9)

$$
\begin{aligned}
|\mathrm{II}| & \leqslant \varepsilon\left|\left\langle\widetilde{G}\left(\cdot, \frac{\tau+t}{\varepsilon}\right), \partial_{t} w(\tau+t)\right\rangle\right| \\
& \leqslant \varepsilon M_{1}\left|\partial_{t} w(\tau+t)\right| \leqslant \varepsilon M_{1} \frac{1}{2}\left(\left|\partial_{t} w(\tau+t)\right|^{2}+1\right) .
\end{aligned}
$$

Интеграл III в (3.10) допускает следующую оценку:

$$
\begin{aligned}
|\mathrm{III}| & =\varepsilon\left|\int_{0}^{t}\left\langle\widetilde{G}\left(\frac{\tau+\tau_{1}}{\varepsilon}\right), \partial_{t}^{2} w\left(\tau+\tau_{1}\right)\right\rangle d \tau_{1}\right| \\
& \leqslant \varepsilon \int_{0}^{t}\left\|\widetilde{G}\left(\cdot, \frac{\tau+\tau_{1}}{\varepsilon}\right)\right\|_{1}\left\|\partial_{t}^{2} w\left(\cdot, \tau+\tau_{1}\right)\right\|_{-1} d \tau_{1} \\
& \leqslant \frac{1}{2} \varepsilon \int_{0}^{t}\left\|\widetilde{G}\left(\cdot, \frac{\tau+\tau_{1}}{\varepsilon}\right)\right\|_{1}^{2} d \tau_{1}+\frac{1}{2} \varepsilon \int_{0}^{t}\left\|\partial_{t}^{2} w\left(\cdot, \tau+\tau_{1}\right)\right\|_{-1}^{2} d \tau_{1} .
\end{aligned}
$$

Подынтегральное выражение в последнем интеграле оценивается из уравнений (3.1) и (2.13):

$$
\begin{aligned}
\left\|\partial_{t}^{2} w\right\|_{-1}^{2} & \leqslant 2\left(\left\|\partial_{t}^{2} u^{\varepsilon}\right\|_{-1}^{2}+\left\|\partial_{t} u^{0}\right\|_{-1}^{2}\right), \\
\left\|\partial_{t}^{2} u^{\varepsilon}\right\|_{-1}^{2} & \leqslant C\left(\left\|u^{\varepsilon}\right\|_{1}^{2}+\left\|u^{\varepsilon}\right\|_{-1}^{2}+\left\|\partial_{t} u^{\varepsilon}\right\|_{-1}^{2}+\left\|f\left(u^{\varepsilon}\right)\right\|_{-1}^{2}+\|\widehat{g}\|_{-1}^{2}\right) .
\end{aligned}
$$

Учитьвая (2.5) при $n \geqslant 3$ (случай $n \leqslant 2$ проще), имеем

$$
\left\|f\left(u^{\varepsilon}\right)\right\|_{-1}^{2} \leqslant C_{1}\left\|f\left(u^{\varepsilon}\right)\right\|_{H}^{2} \leqslant C_{2}\left(\left\|u^{\varepsilon}\right\|_{1}+\left|u^{\varepsilon}\right|+1\right)^{2 n /(n-2)}
$$

и аналогичную оценку для $\left\|f\left(u^{0}\right)\right\|_{-1}^{2}$. Так как

$$
\left\|y^{0}(\tau)\right\|_{E}^{2}=\left\|y^{\varepsilon}(\tau)\right\|_{E}^{2}=\left\|u_{0}\right\|_{1}^{2}+\left|u_{1}\right|^{2} \leqslant R^{2}
$$

TO

$$
\left\|y^{0}(\tau+t)\right\|_{E}^{2} \leqslant C_{3}\left(R^{2}\right), \quad\left\|y^{\varepsilon}(\tau+t)\right\|_{E}^{2} \leqslant C_{3}\left(R^{2}\right) .
$$


Из (3.14)-(3.16) следует, что

$$
\begin{aligned}
\varepsilon \int_{0}^{t}\left\|\partial_{t}^{2} w\left(\cdot, \tau+\tau_{1}\right)\right\|_{-1}^{2} d \tau_{1} \\
\leqslant \varepsilon C_{4}\left(\int_{0}^{t}\left(\left|\partial_{t} u^{\varepsilon}\right|^{2}+\left|\partial_{t} u^{0}\right|^{2}\right) d \tau_{1}+\int_{0}^{t}\left(\left\|u^{\varepsilon}\right\|_{1}^{2}+\left\|u^{0}\right\|_{1}^{2}\right) d \tau_{1}\right. \\
\left.\quad+\int_{0}^{t} C_{5}\left(R^{2}\right) d \tau_{1}+\int_{0}^{t}\left(\left|g^{\varepsilon}\right|^{2}+\left|g^{0}\right|^{2}\right) d \tau_{1}\right) \\
\leqslant \varepsilon C_{6}(t+1), \quad C_{6}=C_{6}\left(R,\left\|g^{\varepsilon}\right\|_{L_{2}^{b}},\left|g^{0}\right|\right) .
\end{aligned}
$$

Из (3.8)-(3.17) следует

$$
\begin{aligned}
\|\widetilde{y}(\tau+t)\|_{E}^{2}:= & \|w(\tau+t)\|_{1}^{2}+\left|\partial_{t} w(\tau+t)\right|^{2} \\
\leqslant & |\mathrm{I}|+|\mathrm{II}|+|\mathrm{III}| \\
\leqslant & C_{1}(R, M) \int_{0}^{t}\left\|\widetilde{y}\left(\tau+\tau_{1}\right)\right\|_{E}^{2} d \tau_{1}+\varepsilon M_{1}\left(\left|\partial_{t} w(\tau+t)\right|^{2}+1\right) \\
& +\varepsilon \frac{1}{2} \int_{0}^{t}\left\|\widetilde{G}\left(\cdot, \frac{\tau+\tau_{1}}{\varepsilon}\right)\right\|_{1}^{2} d \tau_{1}+\frac{1}{2} \varepsilon C_{6}(t+1) .
\end{aligned}
$$

При достаточно малом $\varepsilon$ имеем $\varepsilon M_{1}<\frac{1}{2}$. Отсюда, из $(3.18)$ и $(3.9)$

$$
\|\widetilde{y}(\tau+t)\|_{E}^{2} \leqslant C_{7} \int_{0}^{t}\left\|\widetilde{y}\left(\tau+\tau_{1}\right)\right\|_{E}^{2} d \tau_{1}+\varepsilon C_{8}(t+1) .
$$

Обозначив $\|\widetilde{y}(\tau+t)\|_{E}^{2} /(t+1)=v(t)$, из (3.19) выводим дифференциальное неравенство:

$$
v(t) \leqslant C_{7} \int_{0}^{t} v\left(\tau_{1}\right) d \tau_{1}+\varepsilon C_{8} .
$$

Отсюда по неравенству Гронуола следует, что

$$
v(t) \leqslant \varepsilon C_{8} e^{C_{7} t}
$$

и

$$
\|\widetilde{y}(\tau+t)\|_{E}^{2} \leqslant(t+1) v(t) \leqslant \varepsilon C_{8}(t+1) e^{C_{7} t} \leqslant \varepsilon C_{8} e^{2 \rho t},
$$

где $2 \rho=C_{7}+1$.

Мы доказали следующую теорему.

ТЕОРЕма 3.1. При выполнении условий (3.8) и (3.9) для отклонения $w(\tau+t)=u_{\widehat{g}}(\tau+t)-u_{g^{0}}(\tau+t)$ решений задач Коши (3.1), (3.2) и (2.13), (3.2) (при одинаковых начальных условиях $\left.\left(u_{0}(x), u_{1}(x)\right)\right)$ имеет место оценка (3.20)

$$
\|\widetilde{y}(\tau+t)\|_{E}=\left(\|w(\tau+t)\|_{1}^{2}+\left|\partial_{t} w(\tau+t)\right|^{2}\right)^{1 / 2} \leqslant C \varepsilon^{1 / 2} e^{\rho t},
$$

әде $C=C_{8}\left(M, M_{1}, R\right), \rho=\rho(M, R)$. 
Приведем некоторые примеры функций $g(x, z)$, которые удовлетворяют условияМ (3.8) и (3.9).

a) Пусть $g(x, z)$ - тригонометрический полином по $z$ с рационально независимыми частотами $\left(\alpha_{1}, \ldots, \alpha_{l}\right)=\bar{\alpha}$ :

$$
g(x, z)=a_{0}(x)+\sum_{0<|\bar{k}| \leqslant N} a_{\bar{k}}(x) e^{i(\bar{\alpha}, \bar{k}) z}:=g^{0}(x)+\widetilde{g}(x, z)
$$

Здесь $\bar{k}=\left(k_{1}, \ldots, k_{l}\right) \in \mathbb{Z}^{l} \backslash\{0\},|\bar{k}|=k_{1}+\cdots+k_{l}, x \in \mathbb{T}^{n}, z \in \mathbb{R}$. Предполагается, что $\bar{a}_{\bar{k}}=a_{-\bar{k}}$ для $0<|\bar{k}| \leqslant N$ и $a_{\bar{k}}(x) \in H_{1}=H_{1}\left(\mathbb{T}^{n}\right)$. Очевидно, $a_{0}(x)=g^{0}(x)=$ $\bar{g}(x)$ - усреднение $g(x, t / \varepsilon)$ при $\varepsilon \rightarrow 0+$. В этом случае

$$
\widetilde{G}(x, z)=\sum_{0<\bar{k} \leqslant N} \frac{a_{\bar{k}}(x)}{i(\bar{\alpha}, \bar{k})} e^{i(\bar{\alpha}, \bar{k}) z}, \quad \partial_{z} \widetilde{G}=\widetilde{g}(x, z)=g(x, z)-g^{0}(x) .
$$

Так как $\alpha_{j}$ рационально независимы, то

$$
|(\bar{\alpha}, \bar{k})| \geqslant \delta>0 \quad \forall \bar{k}: 0<|\bar{k}| \leqslant N
$$

Функция $\widetilde{G}$ удовлетворяет условию (3.9), так как

$$
\|\widetilde{G}(\cdot, z)\|_{1} \leqslant C \sum_{0<\bar{k} \leqslant N} \frac{\left\|a_{\bar{k}}(\cdot)\right\|_{1}}{\delta} \leqslant M_{1}
$$

Любая функция $\widehat{g}(x, z) \subset \mathscr{H}(g)$ также имеет вид (3.22), а соответствующая ей функция $\widetilde{G}(x, z)$, очевидно, также удовлетворяет оценке $(3.24)$ с той же константой $M_{1}$.

b) Рассмотрим теперь случай квазипериодической по $z$ внешшей силы $g(x, z)$, т.е.

$$
g(x, z)=\Phi(x, z \bar{\alpha}), \quad \bar{\alpha}=\left(\alpha_{1}, \ldots, \alpha_{l}\right)
$$

где $\Phi(x, \bar{\omega})=\Phi\left(x, \omega_{1}, \ldots, \omega_{l}\right)-2 \pi$-периодическая функция по каждому $\omega_{j}, j=$ $1, \ldots, l$. Для простоты предположим, что $\Phi(x, \bar{\omega}) \in C\left(\mathbb{T}^{n}, \mathbb{T}^{l}\right)$. Обозначим

$$
\widetilde{\Phi}(x, \bar{\omega})=\Phi(x, \bar{\omega})-g^{0}(x), \quad g^{0}(x)=\frac{1}{(2 \pi)^{l}} \int_{\mathbb{T}^{l}} \Phi(x, \bar{\omega}) \mu(d \bar{\omega}),
$$

где $\mu(d \bar{\omega})$ - лебегова мера на $\mathbb{T}^{l}$. Тогда

$$
\widetilde{g}(x, z)=\Phi(x, \bar{\alpha} z)-g^{0}(x)=g(x, z)-g^{0}(x)
$$

Очевидно, $g^{0}(x)$ - усреднение функции $g(x, t / \varepsilon)$ при $\varepsilon \rightarrow 0+$. Предположим, что частоты $\left(\alpha_{1}, \ldots, \alpha_{l}\right)=\bar{\alpha}$ рационально независимы и удовлетворяют следующему диофантову условию:

$$
|(\bar{\alpha}, \bar{k})| \geqslant C_{\bar{\alpha}}|\bar{k}|^{-(l-1+\delta)} \quad \forall \bar{k} \in \mathbb{Z}^{l} \backslash\{0\}, \quad \delta>0
$$


Как известно, неравенство (3.25) имеет место, если $\bar{\alpha}$ принадлежит множеству $\mathbb{R}^{l} \backslash Q^{l}:=S^{l}$, где $Q^{l}$ имеет лебегову меру в $\mathbb{R}^{l}$, равную нулю: $\mu\left(Q^{l}\right)=0$ (см. [13], [14]). Пусть $\bar{\alpha} \in S^{l}$. Разложим функцию $\widetilde{\Phi}$ в ряд Фурье по $\omega_{j}, j=1, \ldots, l$, и положим $\omega_{j}=z \alpha_{j}, z \in \mathbb{R}$. Мы получим

$$
\widetilde{g}(x, z)=\widetilde{\Phi}(x, z \bar{\alpha})=\sum_{\bar{k} \neq 0} a_{\bar{k}}(x) e^{i(\bar{\alpha}, \bar{k}) z}
$$

Аналогично (3.23) имеем

$$
\widetilde{G}(x, z)=\sum_{\bar{k} \neq 0} \frac{a_{\bar{k}}(x)}{i(\bar{\alpha}, \bar{k})} e^{i(\bar{\alpha}, \bar{k}) z}
$$

Если выполнено

$$
\sum_{\bar{k} \neq 0}\left\|a_{\bar{k}}(\cdot)\right\|_{1}|\bar{k}|^{l-1+\delta}<+\infty
$$

то, очевидно,

$$
\|\widetilde{G}(\cdot, z)\|_{1} \leqslant M_{1} \quad \forall z \in \mathbb{R} .
$$

Отметим, что если выполнены оценки (3.28), (3.29) для функций $\widetilde{g}(x, z)$ и $\widetilde{G}(x, z)$, то аналогичные оценки справедливы также для любой функции $\widetilde{\widehat{g}}(x, z)=\widehat{g}-g^{0}(x)$, где $\widehat{g}(x, z) \in \mathscr{H}(g(x, z))$. Точнее, для этих функций $\widetilde{g}(x, z)$ существуют функции $\widetilde{\widehat{G}}$, удовлетворяющие оценке вида $(3.29)$ с той же константой $M_{1}$.

\section{§4. Количественная аппроксимация траекторий, лежащих на глобальном аттракторе $\mathscr{A}_{\varepsilon}$}

Выше был построен равномерный (по $\widehat{g}(x, t / \varepsilon) \in \mathscr{H}(g(x, t / \varepsilon)))$ глобальный аттрактор $\mathscr{A}_{\varepsilon}$ семейства процессов $\left\{U_{\widehat{g}}(t, \tau), \widehat{g}(x, t / \varepsilon):=\widehat{g}^{\varepsilon} \in \mathscr{H}(g(x, t / \varepsilon))\right\}$. Кроме того, был построен глобальный аттрактор $\overline{\mathscr{A}}:=\mathscr{A}_{0}$ усредненного уравнения (2.13). В настоящем параграфе будет построена аппроксимация траекторий $u_{\widehat{g}^{\varepsilon}}(x, t) \in \mathscr{A}_{\varepsilon}$ через траектории $u_{g^{0}}(x, t) \in \mathscr{A}_{0}$ и будет дана оценка отклонений $u_{\widehat{g}}(x, t)$ от $u_{g^{0}}(x, t)\left(g^{0}:=\bar{g}\right.$ - усреднение $g^{\varepsilon}$ при $\left.\varepsilon \rightarrow 0\right)$.

Усредненное уравнение (2.13)

$$
\partial_{t}^{2} u^{0}+\gamma \partial_{t} u^{0}=\Delta u^{0}-\delta^{2} u^{0}-f\left(u^{0}\right)+g^{0}(x), \quad x \in \mathbb{T}^{n},
$$

обладает функцией Ляпунова

$$
\mathscr{L}\left(y^{0}(t)\right):=\mathscr{L}\left(u^{0}(t)\right)=\int_{\mathbb{T}^{n}}\left(\frac{1}{2}\left|\partial_{t} u^{0}\right|^{2}+\frac{1}{2}\left|\nabla u^{0}\right|^{2}+\frac{1}{2} \delta^{2}\left|u^{0}\right|^{2}+F\left(u^{0}\right)-g^{0} u^{0}\right) d x,
$$

$y^{0}(t)=\left(u^{0}(t), \partial_{t} u^{0}(t)\right)$. Функция $\mathscr{L}\left(u^{0}(t)\right)$ строго убывает по $t$ вдоль траекторий $u^{0}(t)$, отличных от стационарных точек $z(x)$. Напомним, что имеет место следующее соотношение:

$$
\mathscr{L}\left(u^{0}(t)\right)-\mathscr{L}\left(u^{0}(\tau)\right)=-\gamma \int_{\tau}^{t}\left|\partial_{t} u^{0}(s)\right|^{2} d s, \quad t \geqslant \tau
$$


где $u^{0}(t)$ - решение уравнения (4.1) (см., например, [4]). Так как уравнение (4.1) автономно, то оно порождает полугруппу $\{S(t), t \geqslant 0\}, S(t-\tau) y^{0}(\tau)=y^{0}(t)$, где $y^{0}(t)=\left(u^{0}(t), \partial_{t} u^{0}(t)\right)$ - решение (4.1). Полугруппа $\{S(t), t \geqslant 0\}$ обладает глобальным аттрактором $\mathscr{A}_{0}$.

Предполагается, что уравнение (4.1) обладает лишь конечным числом стационарных точек $z_{i}(x)$,

$$
\Delta z_{i}(x)-\delta^{2} z_{i}(x)-f\left(z_{i}\right)+g^{0}(x)=0, \quad x \in \mathbb{T}^{n},
$$

$\left\{z_{i}(x), i=1, \ldots, N\right\}:=\mathfrak{N}$. Предполагается, что каждая стационарная точка $z_{i}(x)$ является гиперболической (см. [3]-[5]). Тогда, как известно, аттрактор $\mathscr{A}_{0}$ является объединением полных неустойчивых многообразий $M^{u}\left(z_{i}\right)$, проходящих через эти стационарные точки $z_{i}(x)$ :

$$
\mathscr{A}_{0}=\bigcup_{i=1}^{N} M^{u}\left(z_{i}\right)
$$

(см. [3], [4]). Введем понятие конечномерной составной траектории, лежащей на $\mathscr{A}_{0}$ (cM. [4]).

ОПРЕДЕЛЕНИЕ 4.1. Конечномерной составной траекторией (к.с.т.), лежащей на $\mathscr{A}_{0}$, называется функция $\widetilde{y}(t), \tau \leqslant t<+\infty$, со значениями в $\mathscr{A}_{0}$, обладающая следующими свойствами:

1) существуют такие моменты времени $\tau=t_{0}^{0}<t_{1}^{0}<\cdots<t_{m}^{0}<t_{m+1}^{0}=+\infty$, что $\widetilde{y}(t)$ непрерьвна на полуинтервалах $\left[t_{i}^{0}, t_{i+1}^{0}\right), i=0,1, \ldots, m$;

2 ) значения $\widetilde{y}\left(t_{j} \pm 0\right)$ в точках разрыва $t_{j}^{0}, j=1, \ldots, m$, функции $\widetilde{y}(t)$ лежат в окрестности $\mathscr{O}_{\rho}\left(z^{j}\right)$ одной из стационарных точек $z_{i(j)}=z^{j}, j=1, \ldots, m$;

3) $\widetilde{y}\left(t_{j}^{0}\right)=\widetilde{y}\left(t_{j}^{0}+0\right) \in M^{u}\left(z^{j}\right), \widetilde{y}(t)=S\left(t-t_{j}^{0}\right) \widetilde{y}\left(t_{j}^{0}\right) \forall t \in\left[t_{j}^{0}, t_{j+1}^{0}\right)$; очевидно отсюда следует, что $\widetilde{y}(t) \in M^{u}\left(z^{j}\right) \forall t \in\left[t_{j}^{0}, t_{j+1}^{0}\right), j=1, \ldots, m, m \leqslant N$.

Предположим, что выполнено следующее неравенство:

$$
\left\|y\left(t_{2}\right)-y_{1}\left(t_{2}\right)\right\|_{E} \leqslant C e^{\alpha\left(t_{2}-t_{1}\right)}\left\|y\left(t_{1}\right)-y_{1}\left(t_{1}\right)\right\|_{E}
$$

для любых $t_{1}, t_{2}>0$. Здесь $y(t)=S(t) y(0), y_{1}(t)=S(t) y_{1}(0)$, причем $y(0), y_{1}(0) \in$ $\mathscr{O}_{\eta}\left(\mathscr{A}_{0}\right)$, где $\mathscr{O}_{\eta}\left(\mathscr{A}_{0}\right):=\mathscr{O}_{\eta}-\eta$-окрестность $\mathscr{A}_{0}$ в пространстве $E$. Числа $C$ и $\alpha$ зависят только от $\mathscr{O}_{\eta}$.

Для полугрупшы $\{S(t)\}$, порожденной уравнением (4.1), имеет место неравенство (4.4). Доказательство приведено в [4; гл. 5, §7]. В [4] доказана следующая общая теорема.

TЕОРема 4.1. Пусть полугруппа $\{S(t), t \geqslant 0\}$, действующая в банаховом (или гильбертовом) пространстве E, удовлетворяет следующим условиям:

1) $\{S(t)\}$ обладает глобальнылм $(E, E)$ аттрактором $\mathscr{A}_{0}$;

2) выполнено условие (4.4);

3) существует функиия Ляпунова $\mathscr{L}(y(t))$ nолугруппь $\{S(t)\}$, непрерьвная на $E$;

4) множсество $\mathfrak{N}$ стационарных точек $\{S(t)\}$ конечно: $\mathfrak{N}=\left(z_{1}, \ldots, z_{N}\right)$;

5) nолугруппа $\{S(t)\}-\kappa л а с с а C^{1+\alpha}, 0<\alpha \leqslant 1$, в окрестности каждой точки $z_{i} \in \mathfrak{N}$, и все точки $z_{i}$ гиперболические;

6) $S(t)$ y непрерывна по $(t, y) \in \mathbb{R}_{+} \times E$. 
Пусть $Q$ - компакт в $E, y_{0} \in Q, y(t)=S(t) y_{0}, t \in \mathbb{R}_{+}$.

Тогда для любой такой траектории $y(t)$ полугруппъ $\{S(t)\}$ найдется к.c.m. $\widetilde{y} \in \mathscr{A}_{0}$ (удовлетворяющая всем требованиям определения 4.1) такая, ито имеет место оценка

$$
\|y(t)-\widetilde{y}(t)\|_{E} \leqslant C e^{-\nu t} \quad \forall t \in[0,+\infty),
$$

где $C$ зависит лишь от $Q$, а $\nu>0$ зависит только от $\{S(t)\}$.

Применим теорему 4.1 к уравнению (4.1) или, что эквивалентно, к порожденной ею полугруппе $\{S(t), t \geqslant 0\}$. Предположим дополнительно, что функция взаимодействия $f(u)$ удовлетворяет условию

$$
\left|f^{\prime}(u)-f^{\prime}(v)\right| \leqslant C|u-v|^{\alpha}(1+|u|+|v|)^{\rho}, \quad \alpha+\rho<\frac{2}{n-2}
$$

при $n \geqslant 3$ и $\alpha>0$ и $\rho>0$ ( $\rho$ - любое число) при $n=1,2$.

Тогда, как показано в [4], если функция $f(u)$ удовлетворяет условиям, сформулированным в $\S 2$, и условию (4.6), $\gamma, \delta^{2}>0, g_{0}(x) \in H$, то полугруппа $\{S(t)\}$, порожденная гиперболическим уравнением (4.1), удовлетворяет условиям 1)-6) теоремы 4.1. Следовательно, для каждой ее траектории $\left(u^{0}(t), \partial_{t} u^{0}(t)\right)=y^{0}(t)$ сушествует к.с.т. $\widetilde{y}(t)$ такая, что имеет место (4.5). В силу автономности уравнения (4.1) аналогичная (4.5) оценка справедлива также для траекторий $\{y(t), t \geqslant \tau\}$ этого уравнения при любом $\tau \in \mathbb{R}$ :

$$
\left\|y^{0}(t+\tau)-\widetilde{y}(t+\tau)\right\|_{E} \leqslant C e^{-\nu t} \quad \forall t \in[0,+\infty),
$$

где $\widetilde{y}(t) \in \bigcup_{i=1}^{N} M^{u}\left(z_{i}\right)=\mathscr{A}_{0}, \widetilde{y}(t)-$ к.с.т.

Рассмотрим равномерный глобальньй аттрактор $\mathscr{A}_{\varepsilon}$ гиперболического уравнения

$$
\partial_{t}^{2} u+\gamma \partial_{t} u=\Delta u-\delta^{2} u+f(u)+\widehat{g}\left(x, \frac{t}{\varepsilon}\right), \quad \widehat{g}\left(x, \frac{t}{\varepsilon}\right):=\widehat{g}^{\varepsilon},
$$

где $\widehat{g}^{\varepsilon} \in \mathscr{H}(g(x, t / \varepsilon)):=\mathscr{H}\left(g^{\varepsilon}\right), g(x, t / \varepsilon):=g^{\varepsilon}, \varepsilon-$ фиксированное число, $0<\varepsilon \leqslant$ $\varepsilon_{0}$. Ядро $\mathscr{K}_{\widehat{g}} \varepsilon$ уравнения (4.8) состоит из полных, ограниченных в $E$ траекторий $\left\{y_{\widehat{g}^{\varepsilon}}(t), t \in \mathbb{R},\left\|y_{\widehat{g}^{\varepsilon}}(t)\right\|_{E} \leqslant M_{y_{\widehat{g}^{\varepsilon}}}\right.$, причем $\left.y_{\widehat{g}^{\varepsilon}}(t):=\left(u_{\widehat{g}^{\varepsilon}}(t), \partial_{t} u_{\widehat{g}^{\varepsilon}}(t)\right) \in \mathscr{A}_{\varepsilon}\right\}$. Имеет место следующая формула:

$$
\mathscr{A}_{\varepsilon}=\bigcup_{\widehat{g}^{\varepsilon} \in \mathscr{H}\left(g^{\varepsilon}\right)} \mathscr{K}_{\widehat{g}^{\varepsilon}}(0)=\bigcup_{\widehat{g}^{\varepsilon} \in \mathscr{H}\left(g^{\varepsilon}\right)} \mathscr{K}_{\widehat{g}}(t), \quad t \in \mathbb{R}, \quad t \text { фиксировано, }
$$

где $\mathscr{K}_{\widehat{g}}(t)$ - сечение $\mathscr{K}_{\widehat{g}}$.

Рассмотрим кусок траектории $y_{\widehat{g}^{\varepsilon}}(t):=\widehat{y}^{\varepsilon}(t) \subset \mathscr{A}_{\varepsilon}$ :

$$
\{\widehat{y}(\tau+t),-T \leqslant t \leqslant 0\},
$$

причем $T$ будет определено ниже. Для простоты записи будем сначала считать, что $\tau=0$. Из точки $\widehat{y}(-T)$ выходит траектория $y^{0}(-T+t)=\left(u^{0}(-T+t)\right.$, $\left.\partial_{t} u^{0}(-T+t)\right), t \geqslant 0$, усредненного уравнения (4.1), удовлетворяюшая при $t=0$ условию

$$
\widehat{y}^{\varepsilon}(-T)=y^{0}(-T) \text {. }
$$


Как было показано в $\S 2$, процессы $\left\{U_{\widehat{g}^{\varepsilon}}(t, \tau), \widehat{g}^{\varepsilon} \in \mathscr{H}\left(g^{\varepsilon}\right)\right\}$ обладают компактньп притягиваюшим множеством $[\mathscr{P}] \Subset E$. Поэтому $\mathscr{A}_{\varepsilon} \subset[\mathscr{P}]$ и $y^{0}(-T) \in[\mathscr{P}]$. Так как $y^{0}(-T)$ принадлежит фиксированному компактному множеству [ $\left.\mathscr{P}\right]$, то согласно теореме 4.1 можно найти к.с.т. $\left\{\widetilde{y}^{0}(-T+t), t \geqslant 0\right\} \subset \bigcup_{i=1}^{N} M^{u}\left(z_{i}\right)=\mathscr{A}_{0}$ такую, что имеет место оценка

$$
\left\|y^{0}(-T+t)-\widetilde{y}^{0}(-T+t)\right\|_{E} \leqslant C_{1} e^{-\nu t} \quad \forall t>0,
$$

где $C_{1}=C_{1}([\mathscr{P}])$, а $\nu>0$ и определяется с помошью усредненного уравнения (4.1) (или, что то же, соответствуюшей ему полугруппой $\{S(t), t \geqslant 0\}$ ).

Так как траектории $\left\{\widehat{y}^{\varepsilon}(-T+t), t \geqslant 0\right\}$ и $\left\{y^{0}(-T+t), t \geqslant 0\right\}$ удовлетворяют одинаковым начальным условиям (4.10), то согласно оценке (3.21)

$$
\left\|\widehat{y}^{\varepsilon}(-T+t)-y^{0}(-T+t)\right\|_{E} \leqslant C \varepsilon^{1 / 2} e^{\rho t}, \quad t \geqslant 0 .
$$

Из (4.11) и (4.12) выводим

$$
\begin{aligned}
& \left\|\widehat{y}^{\varepsilon}(-T+t)-\widetilde{y}^{0}(-T+t)\right\|_{E} \\
& \quad \leqslant\left\|\widehat{y}^{\varepsilon}(-T+t)-y^{0}(-T+t)\right\|_{E}+\left\|y^{0}(-T+t)-\widetilde{y}^{0}(-T+t)\right\|_{E} \\
& \quad \leqslant\left(C+C_{1}\right)\left(\varepsilon^{1 / 2} e^{\rho t}+e^{-\nu t}\right) .
\end{aligned}
$$

Ниже из этой оценки выводится ряд следствий.

ТЕОРема 4.2. Пусть функиия $f(u)$ удовлетворяет условиям, сформулированныц в $\S 2$, и условию (4.6); $\gamma>0, \delta^{2}>0 ; g^{0}(x) \in H=L_{2}(\Omega)$; $\widehat{g}(x, z)-g^{0}(x):=\widetilde{g}(x, z)$, әде $\widehat{g}(x, t / \varepsilon)=\widehat{g}^{\varepsilon}(x, t) \in \mathscr{H}\left(g^{\varepsilon}\right) ; \widetilde{g}(x, z)$ удовлетворяет условиям (3.8), (3.9).

Тогда имеет место следующая оценка для отклонения аттракторов $\mathscr{A}_{\varepsilon}$ om $\mathscr{A}_{0}$ :

$$
\operatorname{dist}_{E}\left(\mathscr{A}_{\varepsilon}, \mathscr{A}_{0}\right) \leqslant C \varepsilon^{\nu /(2(\rho+\nu))},
$$

где $\nu$ и $\rho$ определены в формулах (4.11) и (4.12).

ДокаЗАТЕЛЬСТво. Положим в (4.13) $T=T_{\varepsilon}=(1 /(2(\rho+\nu))) \log (1 / \varepsilon)$ и $t=T_{\varepsilon}$. Для такого значения $T_{\varepsilon}$ имеем

$$
e^{-\nu T_{\varepsilon}}=\varepsilon^{1 / 2} e^{\rho T_{\varepsilon}}=\varepsilon^{\nu /(2(\rho+\nu))} .
$$

Из (4.13) получим

$$
\begin{aligned}
& \left\|\widehat{y}^{\varepsilon}\left(-T_{\varepsilon}+T_{\varepsilon}\right)-\widetilde{y}^{0}\left(-T_{\varepsilon}+T_{\varepsilon}\right)\right\|_{E} \\
& \quad=\left\|\widehat{y}^{\varepsilon}(0)-\widetilde{y}^{0}(0)\right\|_{E} \leqslant 2\left(C+C_{1}\right) \varepsilon^{\nu /(2(\rho+\nu))}=C_{2} \varepsilon^{\nu /(2(\rho+\nu))} .
\end{aligned}
$$

Согласно (4.9) $\left.\widehat{y}^{\varepsilon}(t)\right|_{t=0}=\left.\widehat{y}_{\widehat{g}}^{\varepsilon}(t)\right|_{t=0} \forall \widehat{g}^{\varepsilon} \subset \mathscr{H}\left(g^{\varepsilon}\right)-$ любая точка $\mathscr{A}_{\varepsilon}$. Очевидно, $\widetilde{y}^{0}(0) \in \mathscr{A}_{0}$. Поэтому из (4.15) следует (4.14). 
ЗАмЕчАнИЕ 4.1. Оценка (4.14) имеет место при менее ограничительных условиях на функцию $f(u)$. Для краткости изложения мы ограничились функциями $f(u)$, указанньми в теореме 4.2 .

Рассмотрим теперь вопрос об аппроксимизации куска траектории, например, $\left\{\widehat{y}\left(-T_{\varepsilon}+t\right), T_{\varepsilon}-r_{\varkappa}(\varepsilon) \leqslant t \leqslant T_{\varepsilon}\right\} \in \mathscr{A}_{\varepsilon}$ через кусок к.с.т. $\left\{\widetilde{y}^{0}\left(-T_{\varepsilon}+t\right), T_{\varepsilon}-r_{\varkappa}(\varepsilon) \leqslant\right.$ $\left.t \leqslant T_{\varepsilon}\right\} \in \mathscr{A}_{0}=\bigcup_{i=1}^{N} M^{u}\left(z_{i}\right) ; r_{\varkappa}(\varepsilon)>0$. (При этом $T_{\varepsilon}$ можно заменить любым другим числом (см. нижеследуюшее).)

ТЕОрема 4.3. Любой кусок траектории $\widehat{y}^{\varepsilon}(t) \in \mathscr{A}_{\varepsilon}$ временной длинь

$$
r_{\varkappa}(\varepsilon)=\frac{1}{(\rho+\nu)} \frac{\varkappa}{2(2+\varkappa)} \log \frac{1}{\varepsilon}, \quad \varkappa>0
$$

m.e.

$$
\widehat{y}^{\varepsilon}(t) \in \mathscr{A}_{\varepsilon}, \quad \theta \leqslant t \leqslant \theta+r_{\varkappa}(\varepsilon), \quad \theta \in \mathbb{R},
$$

можсно аппроксимировать соответствующей ей к.с.m. $\left\{\widetilde{y}^{0}(t), \theta \leqslant t \leqslant \theta+\right.$ $\left.r_{\varkappa}(\varepsilon)\right\} \in \bigcup_{i=1}^{N} M^{u}\left(z_{i}\right)$, причем на любом $M^{u}\left(z_{i}\right)$ лежит не более одного непрерьвноого куска $\widetilde{y}^{0}(t)$.

Имеет место следующая оценка

$$
\left\|\widehat{y}^{\varepsilon}(t)-\widetilde{y}^{0}(t)\right\|_{E} \leqslant C_{3} \varepsilon^{\nu /((2+\varkappa)(\rho+\nu))},
$$

$\theta \leqslant t \leqslant \theta+r_{\varkappa}(\varepsilon)$.

Константа $C_{3}$ не зависит от $\varkappa, \theta$ и $\widehat{y}^{\varepsilon}(t)$.

ДокАЗАТЕльство. Как показано ниже, оценку (4.17) достаточно доказать для некоторого конкретного начального времени $\theta$. Возьмем сначала

$$
\begin{aligned}
\theta=T_{\varepsilon}-r_{\varkappa}(\varepsilon) & =\frac{1}{2} \frac{1}{\rho+\nu} \log \frac{1}{\varepsilon}-\frac{\varkappa}{2(2+\varkappa)} \frac{1}{\rho+\nu} \log \frac{1}{\varepsilon} \\
& =\frac{1}{2+\varkappa} \frac{1}{\rho+\nu} \log \frac{1}{\varepsilon} .
\end{aligned}
$$

Тогда

$$
\theta+r_{\varkappa}(\varepsilon)=T_{\varepsilon}=\frac{1}{2(\rho+\nu)} \log \frac{1}{\varepsilon} .
$$

Аналогично (4.10) пусть $\left\{y^{0}\left(-T_{\varepsilon}+t\right), t \geqslant 0\right\}-$ траектория усредненного уравнения (4.1), которая при $t=0$ совпадает с $\widehat{y}^{\varepsilon}\left(-T_{\varepsilon}\right)$ :

$$
\widehat{y}^{\varepsilon}\left(-T_{\varepsilon}\right)=y^{0}\left(-T_{\varepsilon}\right)
$$

Тогда, как было показано выше, имеет место оценка $\left(4.12_{\varepsilon}\right)$, совпадающая с $(4.12)$ с заменой $T$ на $T_{\varepsilon}$. Далее, аналогично $(4.10),(4.11)$ для траектории $y^{0}\left(-T_{\varepsilon}+t\right)$ можно найти такую к.с.т. $\left\{\widetilde{y}^{0}\left(-T_{\varepsilon}+t\right), t \geqslant 0\right\} \in \mathscr{A}_{0}=\bigcup_{i=1}^{N} M^{u}\left(z_{i}\right)$, что имеет место оценка $\left(4.11_{\varepsilon}\right)$, совпадающая с $(4.11)$ с заменой $T$ на $T_{\varepsilon}$.

Из оценок $\left(4.12_{\varepsilon}\right)$ и $\left(4.11_{\varepsilon}\right)$ получаем (аналогично $(4.13)$ )

$$
\left\|\widehat{y}^{\varepsilon}\left(-T_{\varepsilon}+t\right)-\widetilde{y}^{0}\left(-T_{\varepsilon}+t\right)\right\|_{E} \leqslant\left(C+C_{1}\right)\left(\varepsilon^{1 / 2} e^{\rho t}+e^{-\nu t}\right) .
$$




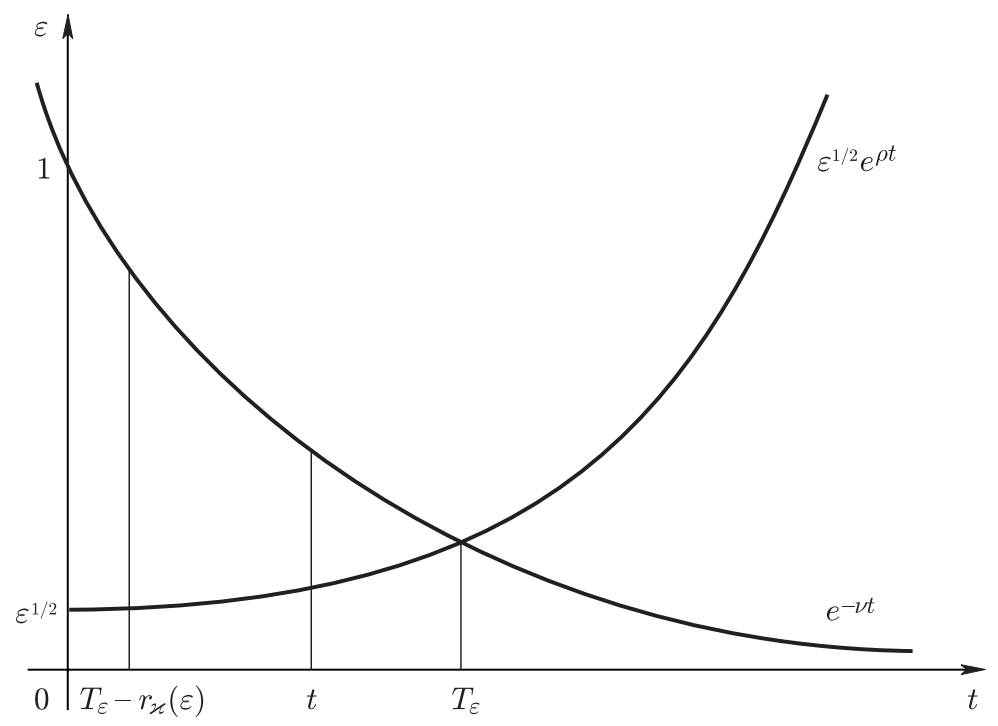

Рис. 1

Из рис. 1 видно, что при

$$
T_{\varepsilon}-r_{\varkappa}(\varepsilon)<t<T_{\varepsilon}
$$

имеем

$$
\varepsilon^{1 / 2} e^{\rho t}<e^{-\nu t}
$$

При $t=T_{\varepsilon}$ имеем $\varepsilon^{1 / 2} e^{\rho t}=e^{-\nu t}$.

Отсюда и из (4.21) следует, что при

$$
\begin{aligned}
T_{\varepsilon} & -r_{\varkappa}(\varepsilon) \leqslant t \leqslant T_{\varepsilon} \\
\left\|\widehat{y}\left(-T_{\varepsilon}+t\right)-\widetilde{y}^{0}\left(-T_{\varepsilon}+t\right)\right\|_{E} & \leqslant\left(C+C_{1}\right)\left(e^{-\nu t}+\varepsilon^{1 / 2} e^{\rho t}\right) \\
& \leqslant 2\left(C+C_{1}\right) e^{-\nu t} \leqslant 2\left(C+C_{1}\right) e^{-\nu\left(T_{\varepsilon}-r_{\varkappa}(\varepsilon)\right)} \\
: & =C_{3} \varepsilon^{\nu /((2+\varkappa)(\rho+\nu))}, \quad C_{3}=2\left(C+C_{1}\right) .
\end{aligned}
$$

При этом мы воспользовались формулой (4.18) для $T_{\varepsilon}-r_{\varkappa}(\varepsilon)$.

Из оценки (4.23) следует оценка (4.17), в которой $\theta \leqslant t \leqslant \theta+r_{\varkappa}(\varepsilon)$. Действительно, (4.23) можно записать в виде

$$
\left\|\widehat{y}^{\varepsilon}\left(t_{1}\right)-y^{0}\left(t_{1}\right)\right\|_{E} \leqslant C_{3} \varepsilon^{\nu /((2+\varkappa)(\rho+\nu))}, \quad-r_{\varkappa}(\varepsilon) \leqslant t_{1} \leqslant 0
$$

Отметим, что выбор начального значения $t_{1}, t_{1}=-r_{\varkappa}(\varepsilon)$, в $(4.24)$ не играет роли.

Действительно, напомним, что в рассматриваемом в $\left(4.9^{\prime}\right)$ куске траектории $\widehat{y}^{\varepsilon}(t)\left(\widehat{y}^{\varepsilon}(\tau+t),-T \leqslant t \leqslant 0\right)$ мы могли взять значение $\tau$ любым (а не как выше $\tau=$ $0)$ и с этим значением $\tau$ продолжить все последующие, начиная с $\left(4.9^{\prime}\right)$, выкладки. Выбрав соответствуюшее значение $\tau$, мы получим в (4.24) временной интервал $\theta \leqslant$ 
$t \leqslant \theta+r_{\varkappa}(\varepsilon), \theta-$ любое число, $\theta \in \mathbb{R}$, указанный в формуле (4.17). Оценка (4.17) установлена.

Отметим, что к.с.т. $y^{0}\left(t_{1}\right)$ в $(4.24)$ состоит из кусков траекторий усредненного уравнения (4.1), причем на любом $M^{u}\left(z_{i}\right)$ лежит не более одного куска.

СлЕДСтвИЕ 4.1. Рассмотрим кусок траектории $\left\{\widehat{y}^{\varepsilon}(t), \theta \leqslant t \leqslant \theta+\right.$ $\left.l r_{\varkappa}(\varepsilon)\right\} \in \mathscr{A}_{\varepsilon}, l \in \mathbb{N}$. Согласно теореме (4.3) для любой части этой траектории вида $\left\{\widehat{y}^{\varepsilon}(t), \theta+i r_{\varkappa}(\varepsilon) \leqslant t<\theta+(i+1) r_{\varkappa}(\varepsilon)\right\}, 0 \leqslant i \leqslant l-1$, существует к.c.m. $\left\{\widetilde{y}_{i}^{0}, \theta+i r_{\varkappa}(\varepsilon) \leqslant t<\theta+(i+1) r_{\varkappa}(\varepsilon)\right\} \subset \mathscr{A}^{0}=\bigcup_{j=1}^{N} M^{u}\left(z_{j}\right)$ такая, что

$$
\left\|\widehat{y}^{\varepsilon}(t)-\widetilde{y}_{i}^{0}(t)\right\|_{E} \leqslant C_{3} \varepsilon^{\nu /((2+\varkappa)(\rho+\nu))}, \quad \theta+i r_{\varkappa}(\varepsilon) \leqslant t<\theta+(i+1) r_{\varkappa}(\varepsilon),
$$

$i=0,1, \ldots, l-1$. Обозначим через $\widetilde{y}^{0}(t, l)$ кусочно непрерывную траекторию усредненного уравнения (4.1), задаваемую формулой

$$
\widetilde{y}^{0}(t, l)=\left\{\widetilde{y}_{i}^{0}(t), \theta+i r_{\varkappa}(\varepsilon) \leqslant t<\theta+(i+1) r_{\varkappa}(\varepsilon), i=0,1, \ldots, l-1\right\} .
$$

Из оченок (4.25) следует

$$
\left\|\widehat{y}^{\varepsilon}(t)-\widetilde{y}^{0}(t, l)\right\|_{E} \leqslant C_{3} \varepsilon^{\nu /((2+\varkappa)(\rho+\nu))}, \quad \theta \leqslant t \leqslant \theta+l r_{\varkappa}(\varepsilon) .
$$

ПРЕДЛОЖЕНИЕ 4.4. Для любого куска траектории $\left\{\widehat{y}^{\varepsilon}(t), \theta \leqslant t \leqslant \theta+\right.$ $\left.l r_{\varkappa}(\varepsilon) \mid\left\{\widehat{y}^{\varepsilon}(t)\right\} \subset \mathscr{A}_{\varepsilon}\right\}, l \in \mathbb{Z}_{+}$, существует такая аппроксимирующая ее кусочно непрерьвная траектория $\left\{\widetilde{y}^{0}(t), \theta \leqslant t \leqslant \theta+\operatorname{lr}_{\varkappa}(\varepsilon)\right\} \subset \mathscr{A}_{0}=$ $\bigcup_{i=1}^{N} M^{u}\left(z_{j}\right)$, что выполнена оченка $(4.26)$.

Очевидно, что с возрастанием $l$ соответственно возрастает число кусков непрерывности $\widetilde{y}^{0}(t, l)$. Каждый из этих кусков лежит, очевидно, на одном из неустойчивых многообразий $M^{u}\left(z_{j}\right), j=1, \ldots, N$.

\section{Список литературы}

1. Chepyzhov V. V., Vishik M.I. Attractors for equations of mathematical physics. Providence, RI: Amer. Math. Soc., 2002. (Amer. Math. Soc. Collog. Publ. V. 49.)

2. Temam $R$. Infinite dimensional dynamical systems in mechanics and physics. New York: Springer-Verlag, 1988. (Appl. Math. Ser. V. 68.)

3. Hale J. Asymptotic behaviour of dissipative systems. Providence, RI: Amer. Math. Soc., 1988.

4. Бабин А. В., Вишик М.И. Аттракторы эволюционных уравнений. М.: Наука, 1989.

5. Raugel G. Global attractors in partial differential equations // Handbook of Dynamical Systems 2 / ed. B. Fiedler. Amsterdam: North-Holland, 2001.

6. Chepyzhov V.V., Vishik M.I., Wendland W. On non-autonomous sine-Gordon type equations with a simple global attractor // Preprint № 2003/01. Stuttgart: Stuttgart Univ., 2003.

7. Вишик М.И., Фидлер Б. Количественное усреднение глобальных аттракторов гиперболических волновых уравнений с быстро осциллирующими коэффициентами // УМН. 2002. T. 47. № 4. С. 75-94.

8. Ильин A.А. Усреднение диссипативных систем с быстро осциллирующими правыми частями // Матем. сб. 1996. Т. 187. № 5. С. 15-58.

9. Вишик М.И., Чепыжов В.В. Усреднение траекторных аттракторов эволюционных уравнений с быстро осциллирующими членами // Матем. сб. 2001. Т. 192. № 1. С. 16-53. 
10. Haraux A. Two remarks on dissipative hyperbolic problems // Nonlinear partial differential equations and their applications. Collège de France. V. 7 / ed. H. Brezis, J.L. Lions. Boston, MA: Pitman, 1985. P. 161-179. (Res. Notes Math. V. 122.)

11. Chepyzhov V. V., Vishik M.I. Attractors of non-autonomous dynamical systems and their dimension // J. Math. Pures Appl. (9). 1994. V. 73. № 3. P. 279-333.

12. Левитан Б. М., Жиков В.В. Почти периодические функции и дифференциальные уравнения. М.: Изд-во МГУ, 1978.

13. Kassels J. W.S. An introduction to Diophantine approximations. Cambridge: Cambridge Univ. Press, 1957.

14. Fiedler B., Vishik M.I. Quantative homogenization of global attractors for reaction-diffusion systems with rapidly oscillating terms // Preprint № A-18-2000. Berlin: Free Univ. Berlin, 2000.

Институт проблем передачи информации РАН, г. Москва

Поступила в редакцию

E-mail: vishik@itp.ru, chep@iitp.ru 